\title{
Ouvrages de protection dans la zone de déferlement de la houle
}

\section{Introduction}

\author{
PAR
}

\author{
R. Tenaud \\ Ingénieur en Chef des Ponts-et-Chaussées \\ Chef du Service Central Technique des Ponts maritimes \\ et des Voies navigeables
}

Lorsque la houle aborde une pente formée par le rivage ou un ouvrage, il vient un moment où les conditions de profondeur nécessaires à sa propagation ne sont plus remplies.

L'énergie de l'onde est alors libérée, plus ou moins brutalement. Le profil de la vague est modifié, elle s'aplatit et les vitesses horizontales de l'eau augmentent considérablement ; il se produit des projections d'eau mélée d'air et des sortes de chocs : c'est le déferlement.

Les énergies en cause sont très importantes. Pour une houle de $6 \mathrm{~m}$ de creux, $150 \mathrm{~m}$ de longueur d'onde et $10 \mathrm{~s}$ de période, par exemple, l'énergie transmise sur une largeur de $1 \mathrm{~km}$ correspond à une puissance de 3,5 millions de $\mathrm{kW}$.

Les ouvrages soumis au déferlement doivent dissiper une grande part de l'énergie de la houle, ils sont donc particulièrement attaqués.

Les problèmes posés par le dimensionnement de ces ouvrages ont pris une grande importance depuis quelques années, car on est amené à construire de plus en plus dans la zone de déferlement de la houle pour la protection des côtes, les prises d'eau et rejets en mer et les ports de plaisance.

Il faut remarquer que si on a établi de longue date des digues en mer pour la protection des ports de commerce, les fonds devant ces digues sont lc plus souvent suffisamment grands pour que la houle attaque ces ouvrages avant de déferler.
La houle avant déferlement a fait l'objet de très nombreuses études théoriques et on dispose d'éléments en général suffisants pour dimensionner les ouvrages exposés à cette houle.

Mais il n'en est pas.de même pour la houle déferlante. Cela tient, d'une part, à la très grande complexité de ce phénomène, qui n'a pu jusqu'à présent faire l'objet d'une description théorique et, d'autre part, au fait que les essais généraux sur modèle réduit sont longs et difficiles, en raison du grand nombre de paramètres à considérer et de la grande sensibilité à la valeur de certains d'entre eux puisqu'il s'agit d'un phénomène d'instabilité.

Les études sur la houle déferlante n'ont vraiment commencé que depuis la fin de la dernière guerre. Après les recherches de M. l'Ingénieur Général Larras, des essais systématiques ont été effectués, essentiellement aux Etats-Unis par le Coastal Engineering Research Center (Weggel, Galvin, Jackson) sur le déferlement des vagues sur les plages inclinées.

Les résultats de ces essais ont été assez dispersés, des formules empiriques dans une gamme très étroite de valeurs ont pu cependant être établies.

Tout récemment, entre 1970 et 1974 , des séries d'essais sur modèle réduit ont ćtć faitcs par la S.O.G.R.E.A.H. pour l'étude de la protection de la nouvelle route littorale de la 
Réunion, qui est très exposée. En effet, la pente des fonds est assez raide $(10 \%)$ et le passage des cyclones à proximité de l'île provoque des houles de grande longueur d'onde qui peuvent déferler juste au pied du talus de la digue de protection.

A cette occasion, il a paru intéressant de faire une synthèse et de voir comment se placent les résultats de ces essais par rapport aux formules proposées actuellement.
La Direction des Ports Martimes et des Voies Navigables vient, d'autre part, de confier au Laboratoire National d'Hydraulique une première série d'essais systématiques, qui est en cours, et compte bien procéder à d'autres essais dans les années à venir pour essayer de préciser les principes de dimensionnement des ouvrages dans la zone de déferlement de la houle.

\section{Liste des notations}

$T \quad$ Période de la houle.

$H_{0}^{\prime} \quad$ Creux (= ou "amplitude") de la houle non réfractée en grande profondeur.

$H_{b} \quad$ Creux (= ou "amplitude") au déferlement.

$L_{0} \quad$ Longueur d'onde de la houle en eau profonde. $\left(L_{0}(\mathrm{~m})=1,56 T^{2}(\mathrm{~s})\right)$.

$L_{b} \quad$ Longueur d'onde de la houle au déferlement.

$e_{b} \quad$ Surcote du niveau moyen par rapport au niveau de l'eau au repos.

$\eta=\frac{e_{b}}{H_{b}} \quad$ Surcote adimensionnelle.

$d_{b} \quad$ Profondeur de déferlement sous le niveau moyen de la mer.

$\beta_{b}=\frac{d_{b}}{H_{b}} \quad$ Profondeur adimensionnelle de déferlement.

$\frac{H_{b}}{H_{0}^{\prime}} \quad$ Indice de déferlement.

$\frac{H_{b}}{L_{b}} \quad$ Cambrure au déferlement.

$\frac{H_{b}}{L_{0}}=\frac{2 \pi H_{b}}{g T^{2}}=\frac{H_{b}}{1,56 T^{2}} \quad$ Indice de cambrure au déferlement.

Cet exposé abordera successivement :

- nos connaissances actuelles sur le déferlement,

- nos connaissances actuelles sur la stabilité des ouvrages en blocs,

- la comparaison avec les essais effectués pour la protection de la route littorale de la Réunion.

\section{I - Rappel des connaissances actuelles sur le déferlement}

Ces rappels se réfèrent aux divers cours français et étrangers de travaux maritimes, à des travaux de Weggel, Galvin, Roald Svee, Yuan Jan et Pang Mou Lin, de Sigurdsson, ainsi qu'à des publications françaises dont on trouvera la liste en annexe. $m \quad$ Pente de la plage.

$x_{a} \quad$ Distance d'approche d'un déferlement plongeant.

$x_{p} \quad$ Distance de plongée d'un déferlement plongeant.

$x_{s} \quad$ Distance d'écrasement d'un déferlement plongeant.

$\tau_{a}=\frac{x_{a}}{H_{b}} \quad$ Distance d'approche adimensionnelle.

$\tau_{p}=\frac{x_{p}}{H_{b}} \quad$ Distance de plongée adimensionnelle.

$\tau_{s}=\frac{x_{s}}{H_{b}} \quad$ Distance d'écrasement adimensionnelle.

$x_{d} \quad$ Distance de la structure au point de déferlement d'une houle.

$x_{d}=\varphi x_{p} \quad$ avec $0 \leqslant \varphi \leqslant 2$.

$\rho_{a} \quad$ Masse volumique d'un bloc.

$\delta$

Indice de stabilité de la formule d'Hudson.

$H \quad$ Houle de projet.

$\alpha \quad$ Angle de la structure avec l'horizontale.

$\sim \quad$ Coefficient de corrélation.

(8) Equation $n^{\circ} 8$.

[8] Référence $n^{\circ} 8$.

\section{1 - DEFINITIONS RELATIVES AU DEFERLEMENT}

\section{1 - Figure 1}

L'amplitude de déferlement $H_{b}$ est la différence entre la cote maximale et minimale de la position du déferlement au cours d'une période.

La profondeur de déferlement $d_{b}$ peut être prise égale à : $d_{t}$ : profondeur sous le creux, $d_{M}$ : profondeur sous le niveau moyen de l'eau,

$d_{s}$ : profondeur sous le niveau de l'eau au repos (compte tenu de la cote de la marée et des surcotes de vent).

En notations normalisées on prend : 


$$
\begin{gathered}
\beta_{b t}=\frac{d_{t}}{H_{b}} \quad \beta_{b m}=\frac{d_{M}}{H_{b}} \quad \beta_{b s}=\frac{d_{s}}{H_{b}} \\
\sigma=\frac{d a_{t}}{H_{b}} \quad \eta=\frac{e_{b}}{H_{b}}
\end{gathered}
$$

$\operatorname{avec} e_{b}=d_{M}-d_{s}$.

$\eta$, surélévation, peut être importante pour des pentes raides : elle est négligeable pour des pentes faibles.

D'après Galvin, pour des pentes de 0,05 à 010

$$
\text { pour des pentes de } 0,20
$$

$$
\eta=0,04
$$$$
\eta=0,08 \text {. }
$$

Dans cette étude, la profondeur et la cambrure seront définies en fonction du niveau moyen du déferlement. On prendra donc:

$$
d_{b}=d_{M} \quad \text { et } \quad \beta_{b}=\beta_{b M} .
$$

\section{2 - Les principaux types de déferlement}

Les vagues approchant d'une plage se déforment habituellement avant de se briser. On distingue trois types principaux de déferlement :

- le déferlement par déversement ou déversant (en anglais spilling) avec apparition d'écume à mi-hauteur de la vague,

- le déferlement gonflant (en anglais surging),

- le déferlement plongeant (en anglais plunging) avec formation de rouleaux.

Le déferlement déversant se produit lorsque la crête de la vague devient instable et qu'elle s'écroule vers la face avant de la vague sous forme d'un front irrégulier mousseux.

Le déferlement plongeant se produit lorsque la crête de la vague se courbe au-dessus de la face avant et tombe à la base de la vague, en emprisonnant une poche d'air et en produisant un bruit sourd et le développement d'un front vertical.

Le déferlement gonflant se produit lorsque la crête de la vague reste intacte tandis que la base et la face avant de la vague, avec des déferlements mineurs, avancent vers la plage (voir figure 2).

On peut distinguer une variation continue dans les types de rouleaux entre les déferlements déversants, plongeants et gonflants. On peut cependant définir une catégorie supplémentaire de déferlements, le déferlement avec effondrement (collapsing) pour décrire un type de déferlement intermédiaire entre le déferlement plongeant et le déferlement gonflant.

Le déferlement avec effondrement se produit lorsque la crête de la vague demeure non brisée et relativement plate tandis que la partie inférieure de la face avant se raidit et tombe en formant une surface irrégulière et turbulente qui glisse le long de la plage sans développer un front.

Des travaux effectués par Galvin [6] à la suite d'essais en Laboratoire ont mis en évidence cette séquence continue de déferlements. Les types de déferlements définis peuvent être considérés comme des phases de cette séquence, et les déferlements réels peuvent être classés avec les types définis les plus proches de cette séquence.

Dans cette séquence, le type de déferlement gonflant est un cas limite lorsque le déferlement diminue vers zéro. Mais, la plupart des déferlements classés comme gonflants impliquent de fait un léger déferlement en pied de vague.

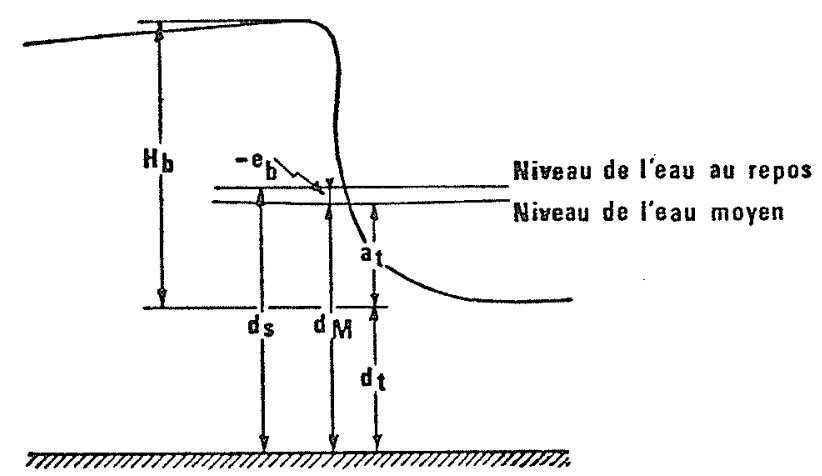

DEFINITION DU DEFERLEMENT

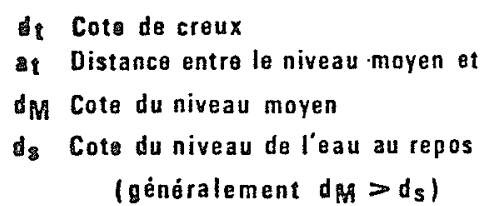

1/
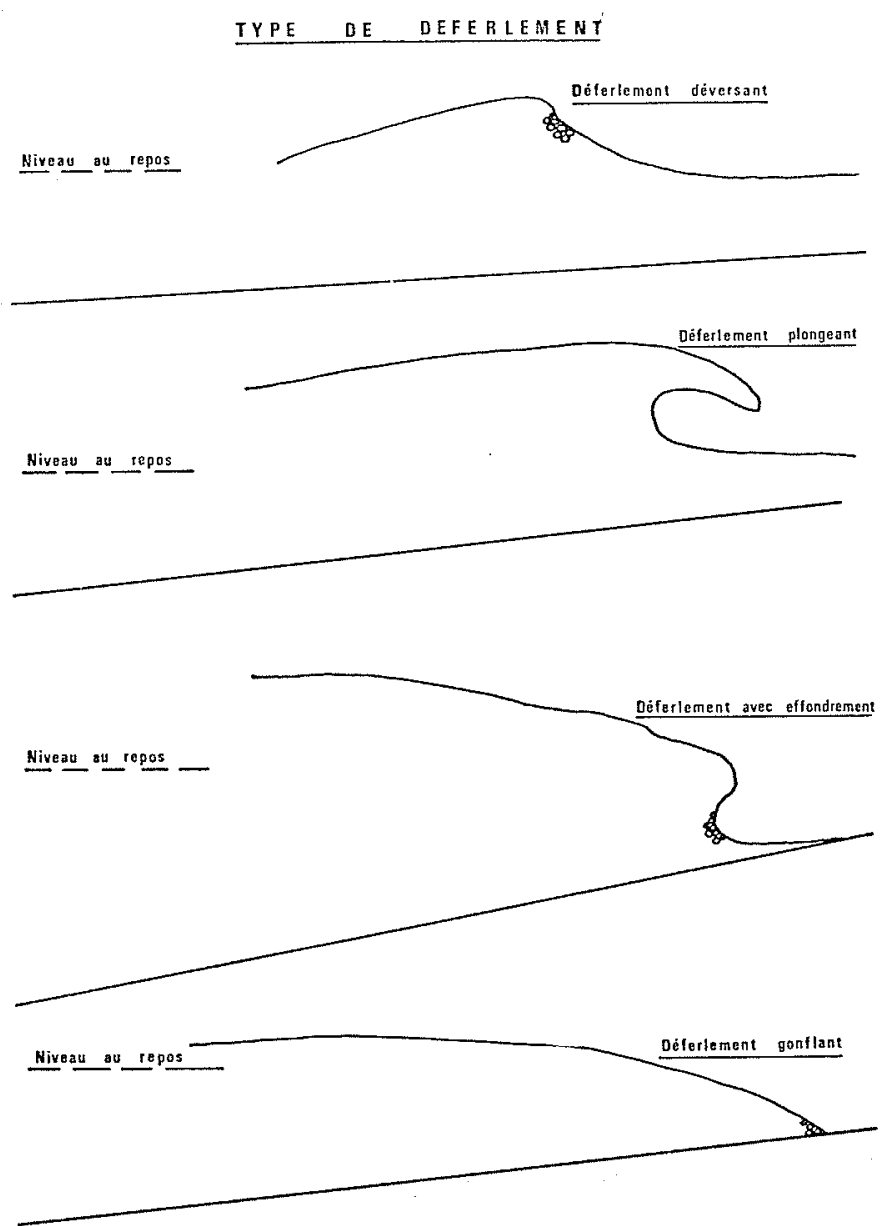


\begin{tabular}{|c|c|c|}
\hline \multicolumn{3}{|c|}{ TYPES DE DEFERLEMENTS IDENTIFIES EN LABORATOIRE } \\
\hline Code & Type de déferlement & Description \\
\hline 1 & Déversant & $\begin{array}{l}\text { Mousse et eau turbulente se produisant sur la face avant. Les } 25 \% \text { supérieurs de } \\
\text { la face avant peuvent devenir verticaux avant de déferler. }\end{array}$ \\
\hline 2 & $\begin{array}{l}\text { Déferlement plongeant bien déve- } \\
\text { loppé }\end{array}$ & $\begin{array}{l}\text { La crête se courbe sur une large poche d'air. Un léger éclatement se produit géné- } \\
\text { ralement. }\end{array}$ \\
\hline 3 & Déferlement plongeant & La crête se courbe moins et la poche d'air est plus petite qu'en 2 . \\
\hline 4 & Déferlement avec effondrement & $\begin{array}{l}\text { Le déferlement se produit sur la moitié inférieure de la vague. Poche d'air mini- } \\
\text { male et généralement pas d'explosion. Bulles et mousses présentes. }\end{array}$ \\
\hline 5 & Déferlement gonflant & $\begin{array}{l}\text { La vague glisse vers le haut de la plage avec peu ou pas de mousse. La surface de } \\
\text { l'eau reste presque plane pendant le reflux sauf là où des ondulations de la plage } \\
\text { peuvent se produire. }\end{array}$ \\
\hline 6 & $\begin{array}{l}\text { Déferlement plongeant altéré par la } \\
\text { vague réfléchie }\end{array}$ & $\begin{array}{l}\text { De petites vagues réfléchies provenant de la vague précédente produisent un pic } \\
\text { sur la crête du déferlement. Le déferlement n'est pas autrement affecté. }\end{array}$ \\
\hline 7 & $\begin{array}{l}\text { Déferlement plongeant altéré par } \\
\text { une vague secondaire }\end{array}$ & $\begin{array}{l}\text { Une première vague peut rattraper une vague immédiatement avant elle, ou, une } \\
\text { vague secondaire immédiatement derrière, en rattrape une précédente, } 1^{\text {er }} \text { cas } \\
\text { difficile à distinguer de } 8 \text {. }\end{array}$ \\
\hline 8 & $\begin{array}{l}\text { Déversement altéré par une vague } \\
\text { secondaire }\end{array}$ & $\begin{array}{l}\text { Une vague secondaire plongeante peut déferler juste devant une vague première } \\
\text { difficile à distinguer de } 7 \text {. }\end{array}$ \\
\hline 9 & Vague secondaire effacée & $\begin{array}{l}\text { La vague en retour d'une vague précédente transporte la vague secondaire au large } \\
\text { où elle peut déferler hors du champ de vision ou simplement disparaitre. }\end{array}$ \\
\hline
\end{tabular}

De même lès déferlements classés dans la catégorie avec effondrement se rapprochent des déferlements plongeants et gonflants.

Sur les plages naturelles, les déferlements les plus couramment observés sont, par fréquence décroissante : les déferlements déversants, les déferlements plongeants, les déferlements avec effondrement, et les déferlements gonflants.

Il est possible que ce phénomène soit dû en partie au fait que les trains de vagues produisant des déferlements gonflants ou avec effondrement peuvent être masqués par des trains de vagues produisant des déferlements plus remarquables : plongeants ou déversants.

Le mode de déferlement ne présente pas seulement un intérêt descriptif ; en effet du mode de déferlement dépend le taux de dissipation d'énergie ou la force instantanée exercée par la vague au déferlement.

Il semblerait que les déferlements les plus dangereux pour les structures soient les déferlements plongeants ou avec effondrement comme on le verra plus loin.

\section{2 - LE DEFERLEMENT DANS UNE MER A PROFONDEUR UNIFORME}

Pour chaque couple : profondeur d'eau et période de la houle, il existe une limite de l'amplitude pour laquelle la houle est stable.

Le critère de déferlement de Stokes pour la houle en profondeur infinie est que la vitesse des particules à la crête de la vague est égale à la célérité de la vague. Si le creux (ou l'am- plitude pour des vagues monochromatiques) était plus important, la vitesse des particules serait supérieure à la célérité et les vagues déferleraient. La condition de Stokes limite l'angle de la crête de la vague au déferlement à $120^{\circ}$.

Michell a exprimé cette condition en terme de cambrure

$$
\left(\frac{H_{b}}{L_{o b}}\right)_{\max }=0,142
$$

où :

$H_{b}$ est le creux de la vague au déferlement, $L_{o b}$ est la longueur d'onde de la houle d'amplitude finie déferlant en eau profonde.

Michell a calculé ainsi le rapport entre cette longueur d'onde en eau profonde et la longueur d'onde $L_{0}$ correspondante de la théorie de la houle de faible amplitude (théorie du $1^{\text {er }}$ ordre).

ou

$$
\begin{gathered}
\frac{L_{o b}}{L_{o}}=1,2 \\
L_{o}=\frac{g T^{2}}{2 \pi}
\end{gathered}
$$

d'où

$$
\frac{H_{b}}{T^{2}}\left(\mathrm{~m} / \mathrm{s}^{2}\right)=0,267
$$

En eau profonde $\left(\frac{h}{L}<\frac{1}{50}\right)$ on utilise comme théorie du $2^{\mathrm{e}}$ ordre la théorie de l'onde solitaire.

Lorsque cette dernière onde se déplace en eau peu profonde le creux de la vague augmente jusqu'à ce qu'elle ne soit plus stable, et déferle. 
Mac Cowan en supposant que cette condition était atteinte lorsque la vitesse des particules d'eau était égale à la célérité de la houle,établit pour l'onde solitaire la relation :

$$
\frac{1}{\beta_{b}}=\left(\frac{H_{b}}{d_{b}}\right)_{\max }=0,78
$$

où $d_{b}$ est la profondeur de déferlement.

Munck [24] en 1949 en déduisait la relation :

$$
\frac{H_{b}}{H_{0}^{\prime}}=\frac{1}{3,3\left(\frac{H_{0}^{\prime}}{L_{0}}\right)^{1 / 3}}
$$

dans laquelle $H_{0}^{\prime}$ est le creux non réfracté en eau profonde.

A l'aide de ces deux critères, Reid et Breitschneider ont établi une courbe de l'indice de déferlement tangente à la droite de l'onde solitaire pour les faibles valeurs de $d_{b}$ et à la droite de Michell pour les grandes profondeurs. Des données empiriques leur permirent de tracer la partie centrale de la courbe (voir figure 4).

Miche [17] a déterminé dans le cas d'une houle en profondeur finie, la forme approchée des solutions correspondant à cette forme limite de la houle. Il a trouvé que cette relation avec la profondeur pouvait être approchée par:

$$
\frac{H_{b}}{L_{b}}=0,142 \text { th }\left(\frac{2 \pi d_{b}}{L_{b}}\right)
$$

où $L_{b}$ est la longueur d'onde au déferlement.

$$
\text { Lorsque : } \frac{d_{b}}{L_{b}} \rightarrow 0, \quad \text { th }\left(\frac{2 \pi d_{b}}{L_{b}}\right) \sim \frac{2 \pi d_{b}}{L_{b}}
$$

donc :

$$
\frac{H_{b}}{d_{b}}=\frac{1}{\beta_{b}} \simeq 0,89
$$

\section{3 - INSUFFISANCE DE LA METHODE TRADITIONNELLE DE CHOIX D'UNE HOULE DE PROJET}

Toute étude de structure côtière commence par la détermiration d'une houle de projet.

Le creux de la houle de projet est le creux de la houle la plus dangereuse, celle exerçant l'effort maximal, sur une structure.

On considère généralement que cet effort maximal est dans le cas de houle non déferlante, celui de la plus grande houle à atteindre la structure, dans le cas d'une houle déferlante celui de la plus grande vague à déferler directement sur la structure.

La profondeur au pied de la structure est calculée d'après les renseignements bathymétriques, la marée et les surcotes de tempête éventuelles.

La hauteur de déferlement correspondante est traditionnellement calculée d'après (6)

soit :

$$
\frac{H_{b}}{d_{b}}=0,78
$$

$$
\beta_{b}=1,28
$$

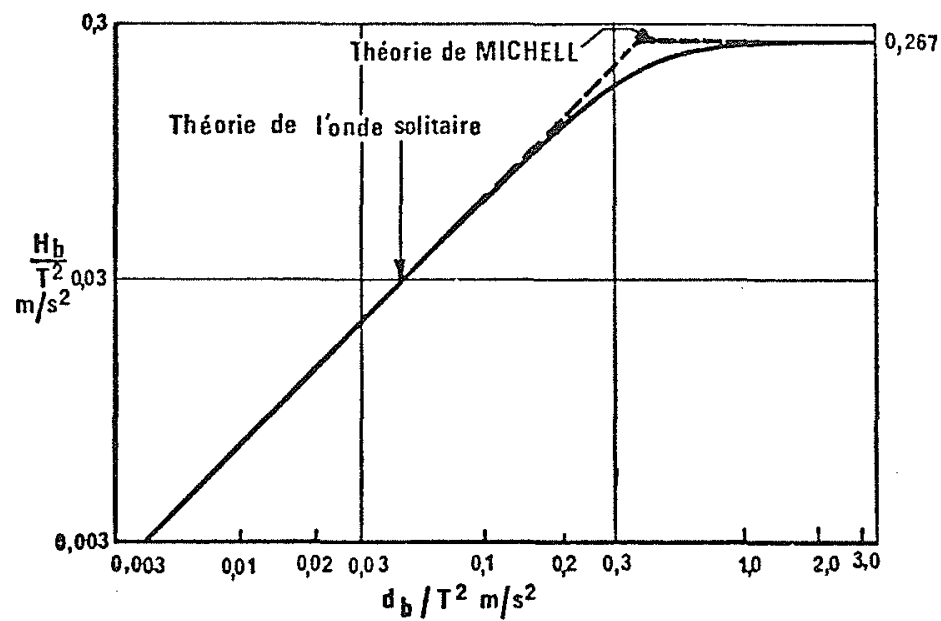

\section{4/ Courbe des indices de déferlement ( $D$ 'après Reid et Breitschneider).}

Pour des sites en mer ouverte, en eau relativement peu profonde $(d<3 \mathrm{~m})$ où il est nécessaire de se protéger contre la maximum absolu du creux de la vague, $H_{b}$ défini par (6) est généralement adopté comme houle de projet.

Pour d'autres sites on tient compte de considérations probabilistes.

Si la hauteur donnée par l'equation (6) se produit plus d'une à deux fois au cours de la vie future de la structure, alors on choisit la houle de creux définie par l'équation (6) comme houle de projet.

Si cette houle ne se produit pas avec une fréquence suffisante, alors la hauteur de la houle de projet est choisie inférieure à la houle précédente d'après la courbe cumulative des fréquences. On choisit alors un lieu déduit de la courbe cumulative des amplitudes moyennes, $H^{1}, H^{1 / 3}$, ou $H^{1 / 10}$ selon le type de structure (flexible, semi-flexible ou rigide).

Cette méthode traditionnelle est cependant l'objet de critiques.

- On suppose que les houles périodiques se conduisent près du déferlement comme des ondes solitaires. Ceci est contesté par certains. En outre des travaux de Lenau [14] aboutissent à une valeur de $\beta_{b}$ voisine de 1,20 pour l'onde solitaire.

- Pour chacune de ces valeurs de $\beta_{b}$ la profondeur appropriée est la profondeur sous le creux $d_{t}$ (Cf. figure 1). Or, on utilise généralement la profondeur au-dessous du niveau de l'eau au repos qui est supérieure. Ce choix compense en général l'erreur commise en surestimant $\beta_{b}$.

- En outre, $\beta_{b}$ est supposé indépendant de la cambrure et de la pente de la plage, ce qui n'est pas exact.

\section{4 - DEFERLEMENT SUR UNE PLAGE EN PENTE}

4.1 - Les théories mathématiques applicables aux houles en profondeur constante sont en principe applicables aux houles sur des fonds en pente, en procédant à la manière de Stokespour les houles d'amplitude finie.

Néanmoins, la détermination de la fonction de potentiel est un problème très difficile qui n'a pu être résolu à notre connaissance que dans des cas particuliers, par exemple en supposant constante la longueur d'onde. 


\section{CLASSIFICATION DES DEFERLEMENTS SELON LA PENTE DE LA PLAGE ET LA CAMBRURE EN EAU PROFONDE}

\begin{tabular}{|c|c|c|c|}
\hline Pente de la plage (m) & Déferlements gonflants & Déferlements plongeants & Déferlements déversants \\
\hline 0,02 & $\frac{H_{0}^{\prime}}{L_{0}} \leqslant 0,0039$ & $0,0039 \leqslant \frac{H_{0}^{\prime}}{L_{0}} \leqslant 0,020$ & $\frac{H_{0}^{\prime}}{L_{0}} \geqslant 0,020$ \\
\hline 0,05 & $\frac{H_{0}^{\prime}}{L_{0}} \leqslant 0,0079$ & $0,0079<\frac{H_{0}^{\prime}}{L_{0}}<0,035$ & $\frac{H_{0}^{\prime}}{L_{0}} \geqslant 0,035$ \\
\hline 0,10 & $\frac{H_{0}^{\prime}}{L_{0}} \leqslant 0,009$ à 0,010 & $0,009 \leqslant \frac{H_{0}^{\prime}}{L_{0}} \leqslant 0,060$ & $\frac{H_{0}^{\prime}}{L_{0}} \geqslant 0,060$ \\
\hline
\end{tabular}

De même, les théories existantes étudient les vagues soit lorsqu'elles approchent du déferlement, soit lorsqu'elles ont déferlé, soit lorsque les conditions initiales ne provoquent pas de déferlement.

Il y a peu d'études théoriques sur la structure du processus de déferlement, malgré les résultats prometteurs obtenus par Harlow et Welch en 1965 [15] et Harlow en 1966 [16].

La seule méthode pratiquement accessible pour l'étude des déferlements est donc actuellement la méthode empirique.

4.2 - Classification des déferlements selon la pente de la plage et la cambrure de la houle.

Par des études systématiques Collins et Wier [18], Galvin [5] et [6], Iversen [19] et [20], Weggel et Maxwell [21] ont trouvé que la géométrie du déferlement dépendait également de la pente de la plage $m$.

Iversen a trouvé que pour des vagues en eau profonde identiques, les déferlements de plus grande amplitude se produisaient sur les plages aux pentes les plus fortes.

En outre, on a observé que l'indice de déferlement $H_{b} / H_{0}^{\prime}$ ( $H_{0}^{\prime}$ étant l'amplitude de la vague non réfractée en eau profonde) diminue avec la cambrure de la vague incidente.

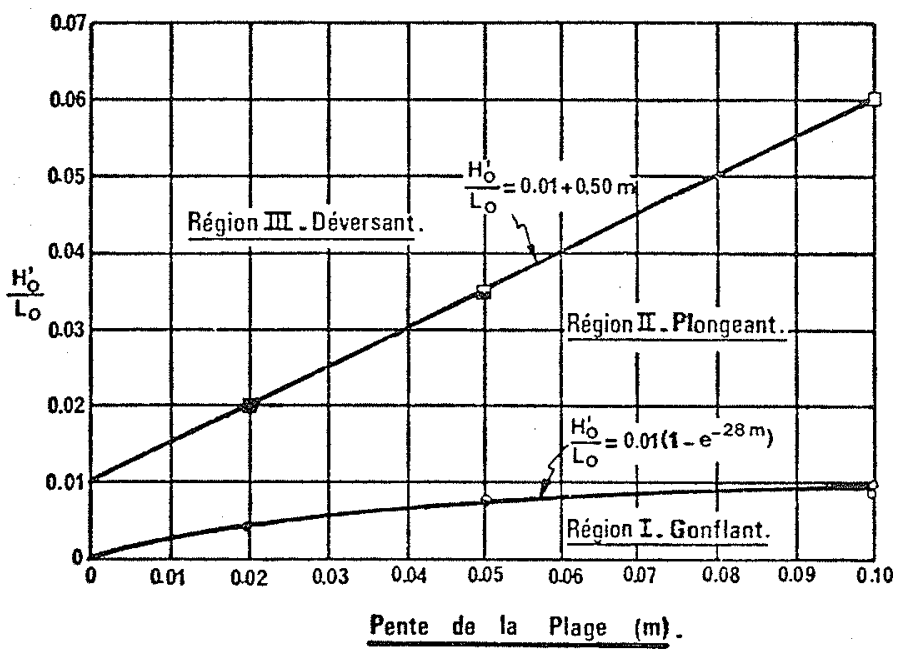

6/ Classement des déferlements selon la pente de la plage et la cambrure en eau profonde ( $D$ 'après Weggel)
La gamme de cambrure pour laquelle un type particulier de déferlement peut être attendu a été défini par Patrick et Wiegel [22] pour des pentes de plages de 0,$1 ; 0,05 ; 0,02$. En général, pour une pente de plage douce, lorsque la cambrure de la vague incidente, non réfractée, en eau profonde augmente, le déferlement fait une transition du gonflant, au plongeant et au déversant.

Etant donnée la présence de déferlement avec effondrement à la limite des déferlements plongeants et déversants, la limite inférieure de la cambrure des déferlements plongeants adoptée ici est celle pour laquelle des déferlements plongeants peuvent être observés.

Le tableau étendu à d'autres valeurs est présenté sous forme de plan (voir figure 6) ; le plan est ainsi partagé en trois régions relatives aux déferlements déversants, plongeants et gonflants.

Ainsi, la pente de la plage et la cambrure de la houle non réfractée en eau profonde déterminent le type de déferlement.

4.3 - Indice de hauteur d'un déferlement en fonction de la cambrure de la houle en eau profonde et de la pente.

On peut rappeler les courbes empiriques de Goda [23] reliant l'indice de hauteur du déferlement $H_{b} / H_{0}^{\prime}$ à la pente de la plage en fonction de la cambrure (fïgure 7).

Sur cette figure on distingue les trois zones de déferlement gonflant, plongeant et déversant.

Les lignes séparatrices sont :

la courbe séparant la région I, déferlements gonflants, de la région II, déferlements plongeants, peut ètre approchée par:

$$
\frac{H_{b}}{H_{0}^{\prime}}=1,53+18,5 \frac{H_{0}^{\prime}}{L_{0}}
$$

- la courbe séparant la région II de la région III, déferlements déversants, peut être approchée par :

$$
\frac{H_{b}}{H_{0}^{\prime}}=0,98+2 \frac{H_{0}^{\prime}}{L_{0}}
$$

On peut rapprocher ces résultats empiriques de la théorie de l'onde solitaire.

On a vu que Minck a établi la relation (7) :

$$
\frac{H_{b}}{H_{0}^{\prime}}=\frac{1}{3,3\left(\frac{H_{0}^{\prime}}{L_{0}}\right)^{1 / 3}}
$$




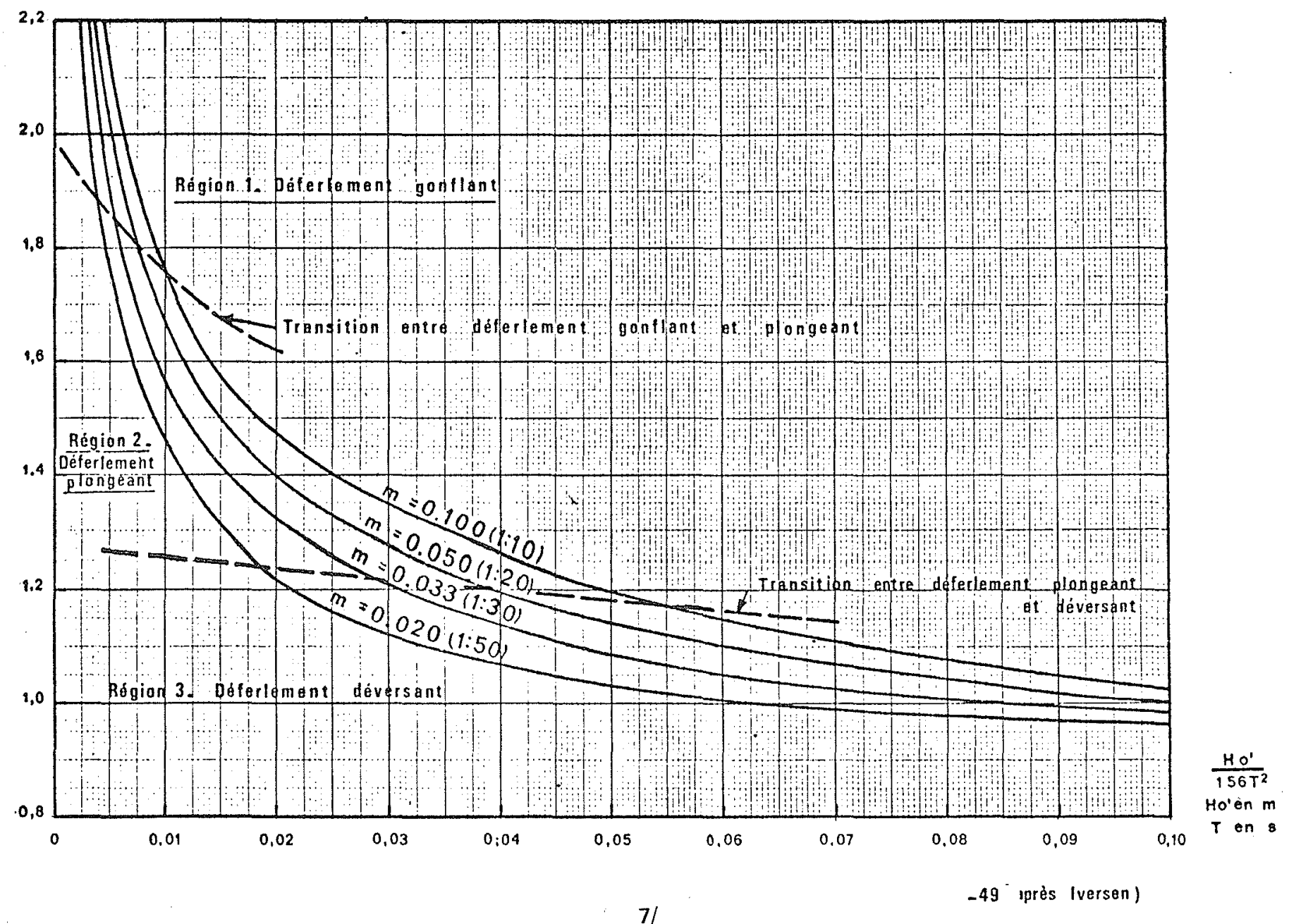

(7) ne tenant pas compte de la pente de la plage, Weggel [8] a recherché dans le cas d'une plage en pente une relation de la forme :

$$
\frac{H_{b}}{H_{0}^{\prime}}=\frac{F(\mathrm{~m})}{\left(\frac{H_{0}^{\prime}}{L_{0}}\right)^{1 / 3}}+G(\mathrm{~m})
$$

dans lesquels $F$ et $G$ sont des fonctions de $m$.

En ajustant cette fonction sur des valeurs de la figure (7) on obtient :

$$
\begin{aligned}
& G(\mathrm{~m})=\frac{D_{1}(1+\mathrm{m})-D_{2}\left(1,715-0,185 e^{-28 \mathrm{~m}}\right)}{D_{1}-D_{2}} \\
& F(\mathrm{~m})=D_{1}[1+\mathrm{m}-G(\mathrm{~m})]
\end{aligned}
$$

avec :

et :

$$
D_{1}=(0,01+0,5 \mathrm{~m})^{1 / 3}
$$

$$
D_{2}=\left(0,01-0,01 e^{-28 m}\right)^{1 / 3}
$$

(13) peut s'écrire $\left(13^{\prime}\right)$ :

$$
\frac{H_{b}}{H_{0}^{\prime}}=\frac{F(\mathrm{~m})\left(\frac{H_{b}}{H_{0}^{\prime}}\right)^{1 / 3}}{\left(\frac{H_{b}}{L_{0}}\right)^{1 / 3}}+G(\mathrm{~m})
$$

Cette famille de courbe a été représentée sur la figure 8.

Les courbes de ces fonctions sont en bon accord avec les travaux d'Iversen.

On doit garder à l'esprit la méthode ayant conduit aux équations 12 à 16 et la dispersion des essais qui ont conduit à elles.

\section{4 - Evaluation du type de déferlement}

Les équations de la figure 6 peuvent nous permettre de définir un critère de type de déferlement en fonction des paramètres définissant les caractéristiques de la cambrure de la houle au large $H_{0}^{\prime} / L_{0}$ ou au rivage $H_{b} / g T^{2}$.

Ces paramètres sont donnés dans le tableau suivant (figure 9).

\section{5 - TRAJET D'UN DEFERLEMENT}

Comme on l'a vu, les déferlements avec effondrement et les déferlements plongeants se produisant en eau peu profonde, sont parmi les plus dangereux pour les structures portuaires. 
5.1 - Description d'un déferlement plongeant

Galvin s'est penché sur les étapes d'un déferlement plongeant entre le moment où la forme de la vague à l'approche du rivage commence à subir d'importantes modifications et le moment où elle s'est complètement écrasée au sol.

Lors d'un déferlement plongeant (cf. figure 10), dans un premier temps la crête de la vague se déplace plus rapidement que la vague jusqu'à ce que la face avant devienne verticale. La crête de la vague tombe alors en se projetant vers l'avant.

Cette crête emprisonne un paquet d'air et frappe ensuite la base de la vague. Elle produit alors des jets d'eau dans toutes directions. Des observations indiquent qu'au moment où ces jets retombent à terre le déferlement principal a eu lieu. La vague poursuit alors jusqu'à sa limite d'ascension.

On peut distinguer théoriquement en laboratoire 4 points .

1) Le point où la vague devient irréversiblement instable.

2) La position observée du déferlement définie comme le lieu où un segment de la face avant de la vague devient vertical.

3) Le point où la crête touche la base de la vague.

4) Le point où les jets d'eau touchent terre.

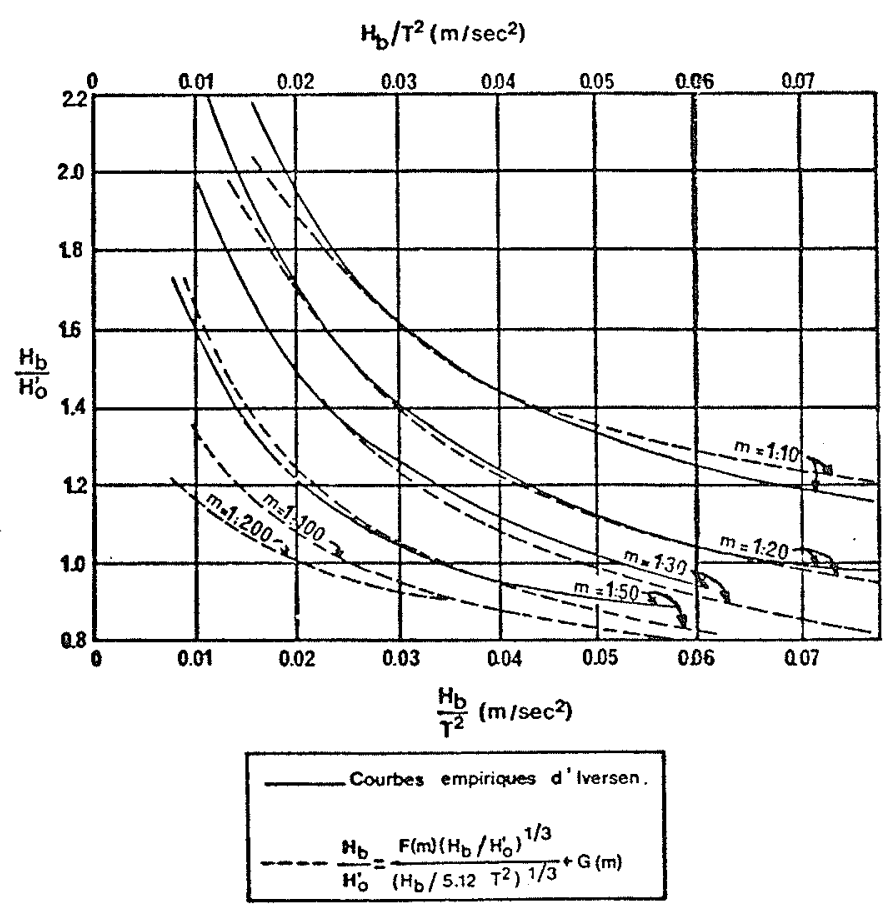

8/ Indice de hauteur de déferlement en fonction de la cambrure du déferlement $\frac{H_{b}}{T^{2}}\left(\mathrm{~m} / \mathrm{sec}^{2}\right)$. (d'après Weggel).

\section{CLASSIFICATION DES DEFERLEMENTS EN FONCTION DE LA CAMBRURE AU LARGE ET A TERRE}

\begin{tabular}{|c|c|c|c|c|c|}
\hline $\begin{array}{c}\text { Paramètre } \\
\text { de } \\
\text { cambrure }\end{array}$ & $\begin{array}{c}\text { Déferlement } \\
\text { gonflant }\end{array}$ & Transition & $\begin{array}{c}\text { Déferlement } \\
\text { plongeant }\end{array}$ & $\begin{array}{c}\text { Transition } \\
\text { déversant }\end{array}$ \\
\hline au large & $\frac{H_{0}^{\prime}}{L_{0}}=\frac{2 \pi H_{0}^{\prime}}{g T^{2}} \leqslant$ & $0,01\left(1-e^{-28 \mathrm{~m}}\right)$ & $\leqslant \frac{H_{0}^{\prime}}{L_{0}} \leqslant \frac{H_{0}^{\prime}}{L_{0}}$ \\
à terre & $\frac{H_{b}}{g T^{2}} \leqslant$ & $\frac{1}{2 \pi}\left(0,0171-0,0190 e^{-28 \mathrm{~m}}\right.$ & $\leqslant \frac{H_{b}}{g T^{2}} \leqslant$ & $0,00159+0,0812 \mathrm{~m}$ \\
$\left.+0,00185 e^{-56 \mathrm{~m}}\right)$ & $+0,0796 \mathrm{~m}^{2}$ & $0,50 \mathrm{~m}$ \\
\hline
\end{tabular}

On appelle les distances entre ces points :

- distance d'approche $x_{a}$;

- distance de plongée $x_{p}$;

- distance d'écrasement $x_{s}$.

En données adimensionnelles

$$
\tau_{a}=\frac{x_{a}}{H_{b}} \quad \tau_{p}=\frac{x_{p}}{H_{b}} \quad \tau_{s}=\frac{x_{s}}{H_{b}}
$$

Distance de déferlement adimensionnelle $=\tau_{p}+\tau_{s}$

\section{2 - Distance d'approche}

Galvin a trouvé :

$$
\begin{array}{llll}
\tau_{a}=\frac{0,48}{\mathrm{~m}} & \text { pour } & \mathrm{m} \geqslant 0,07 \\
\tau_{a}=6,85 & \text { pour } & \mathrm{m}<0,07
\end{array}
$$

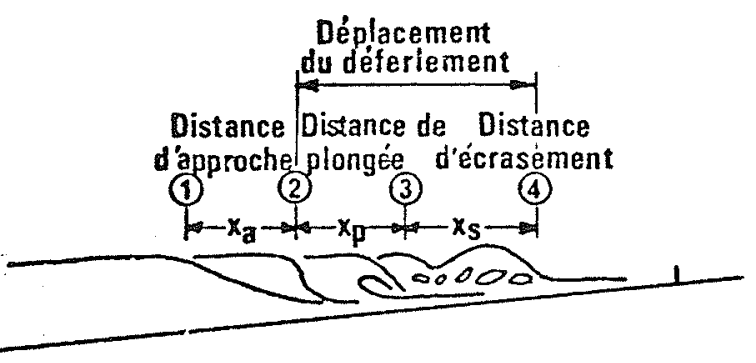

(1) Point d'instabilité initiale

(2) Position de déferlement

(3) Position oú la crète touche la plage

(a) Position où la totalité de la vague touche le sol

10/ Déplacement d'un déferlement plongeant. 


\section{DISTANCE DE PLONGÉE POUR DEUX TYPES DE VAGUES PLONGEANTES}

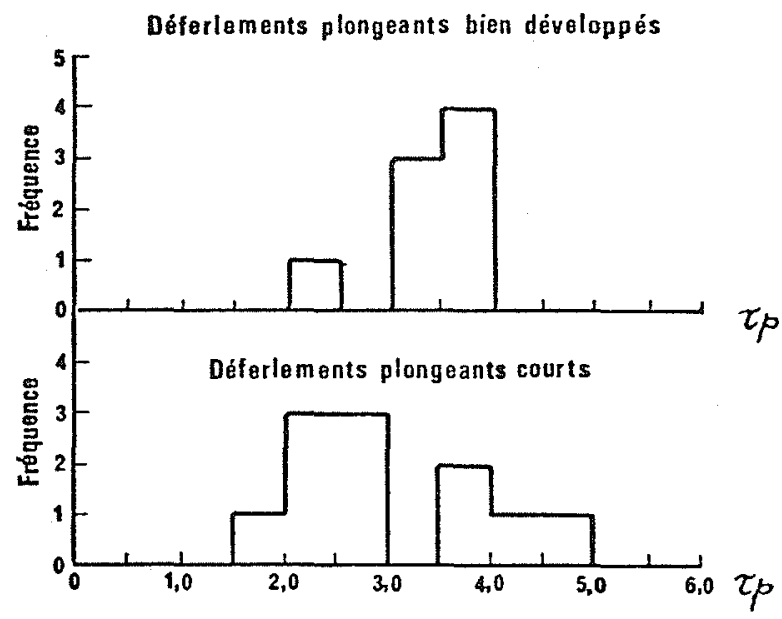

DISTANCE DE PLONGEE ADIMENSIONRELLE

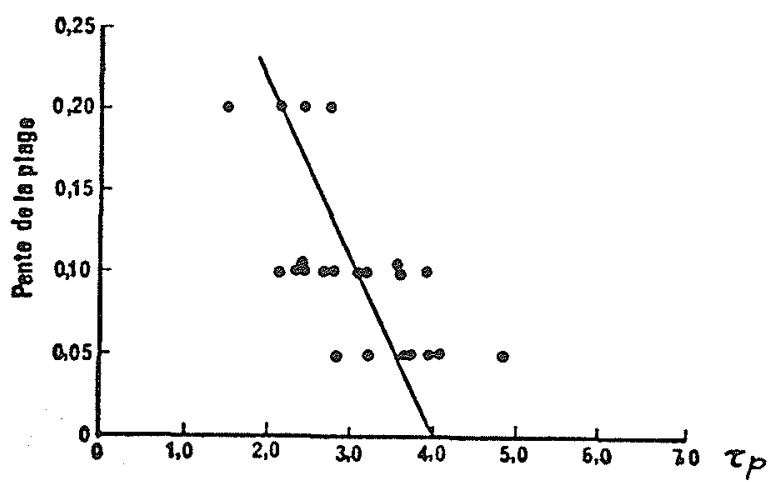
DISTANCE DE PLONGÉE EN FONCTION DE LA
PENTE DE LA PLAGE (D'aprás C.J. GALYIN)

$11 /$

\section{3 - Distance de plongée}

La distance de plongée est de l'ordre de 3 fois l'amplitude au déferlement $H_{b}$ soit $\tau_{p} \sim 3$.

D'après des essais réalisés, il semblerait que les déferlements plongeants bien développés se déplacent habituellement plus loin, horizontalement, avant de toucher le sol, que ne le font les déferlements ordinaires (cf. figure 11).

En fait, la distance de plongée décroît avec la pente et on a pu ajuster la formule suivante avec une assez grande dispersion (cf. figure 11)

$$
\tau_{p}=4-9,25 \mathrm{~m}
$$

Cette formule semblerait être vérifiée même dans le cas d'une pente nulle (Galvin cite des essais où $\tau_{p} \simeq 4$ pour $\mathrm{m}=0$ ).

\section{4 - Distance d'écrasement}

La dissipation d'énergie pendant le processus de déferlement est continue tout au cours de l'écrasement de déferlement celui-ci n'étant complet que lorsque le corps de la vague a atteint le point d'écrasement en frappant le sol (figure 12).

On peut admettre que $\tau_{s}=\tau_{p}$ (voir figure 12).

Dans ces conditions la distance adimensionnelle parcourue par le déferlement devient égale à :

$$
\frac{x_{p}+x_{s}}{H_{b}}=2 \tau_{p}
$$

\section{5 - Conséquences sur le choix d'une houle de projet}

$\beta_{b}$ et $\tau_{p}$ ont leur valeur maximum pour des pentes nulles. Les valeurs ci-dessus de $\tau_{p}$ sont prudentes, la valeur maximum de $\beta_{b}$ ne l'est pas et conduit donc à sous-estimer le creux de déferlement.

Comme par ailleurs le déferlement se poursuit continûment tout au long du parcours, en parcourant la distance d'écrasement la lame possèdera encore une énergie de déferlement considérable. Une procédure prudente consiste donc à localiser les structures au-delà du point d'écrasement.

C'est précisément le cas où une telle localisation n'est pas possible, que nous nous proposons de traiter ici.

\section{6 - AMPLITUDE MAXIMALE DE LA HOULE AU DEFER- LEMENT}

Supposons une structure en mer située sur une pente plane (figure 13).

Pour obtenir une expression de la hauteur maximale de déferlement à laquelle cette structure est soumise, il est néces-

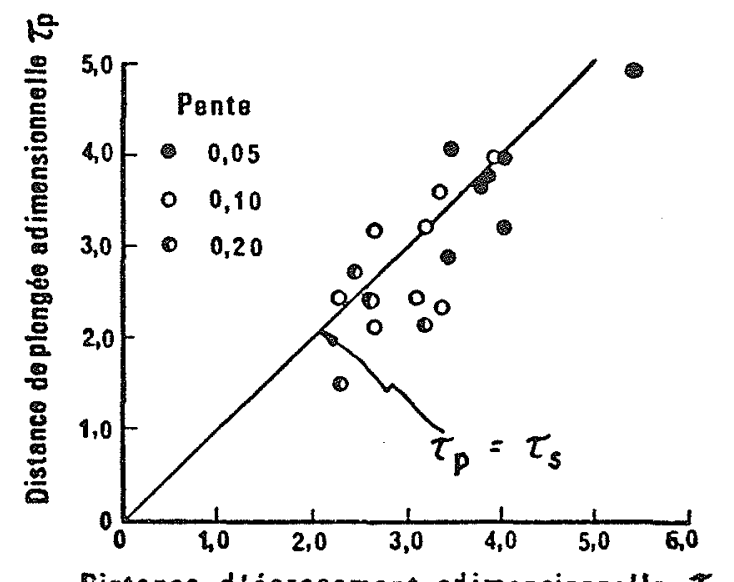

Distanco d'ecrasement adimensionnelle $\tau_{s}$

12/ Distance d'écrasement en fonction de la distance de plongée ( $D^{\prime}$ après C.J. Galvin). 


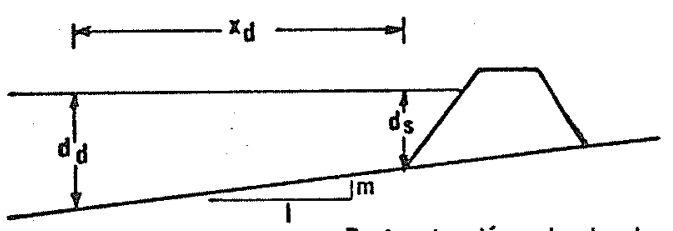

Recherche d'une houle de projet

STRUCTURE SOUMISE AU DEFERLEMEAT

$x_{d}=\varphi x_{p}$

$0.5<\varphi<2$

$13 /$

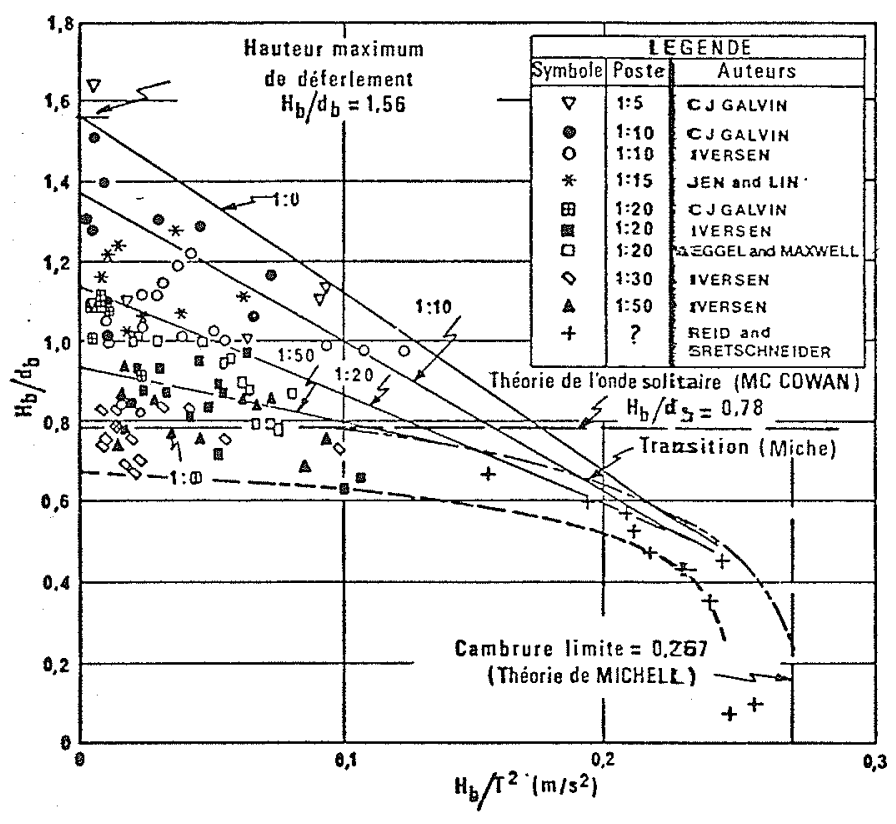

OBSERVATIONS EXPERIMENTALES DE $H_{b} / d_{b}$ EN FONCTION DE LA CAMBRURE AU DEFERLEMENT $\mathrm{H}_{\mathrm{b}} / \mathrm{T}^{2}$

$10^{\prime}$ après Weggel)

14/

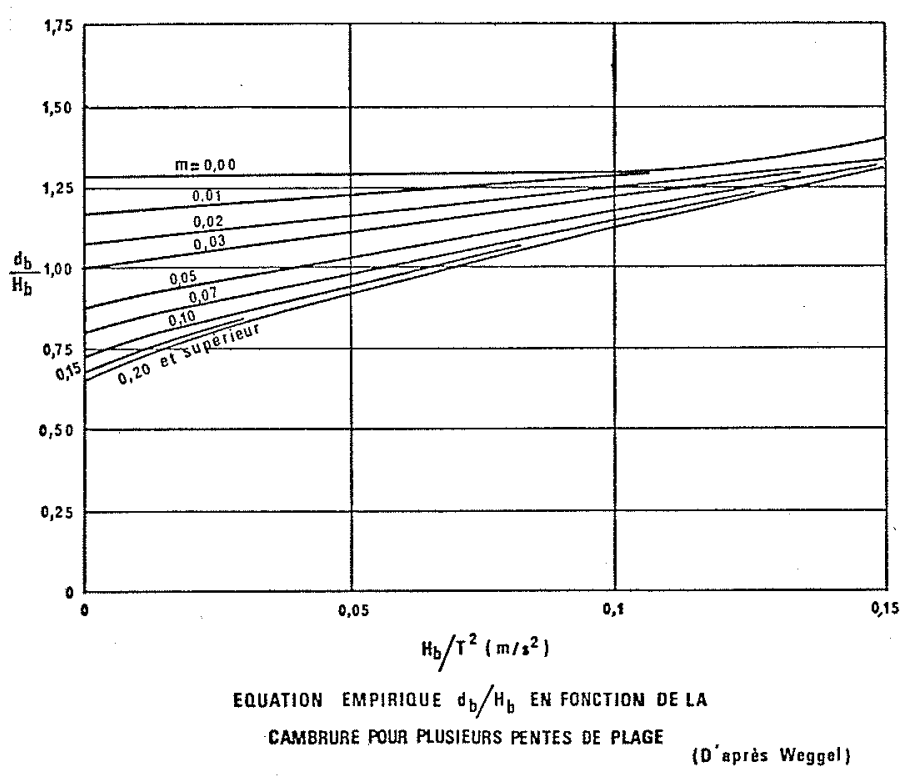

15/ saire de trouver une relation entre la hauteur du déferlement $H_{b}$, la profondeur d'eau au déferlement $d_{b}$, la pente de la plage, la cambrure de la houle et la profondeur au pied de la structure.

Les valeurs données à la figure 14 sont des valeurs expérimentales de $H_{b} / d_{b}$ en fonction de $H_{b} / T^{2}$ pour diverses pentes de plage.

Les équations (5) et (6) sont également représentées sur cette planche ainsi que les transitions données par Reid et Bretschneider [25].

Malgré la dispersion des résultats, il apparaît que $H_{b} / d_{b}$ est une fonction décroissante de la cambrure.

A cambrure constante, $H_{b} / d_{b}$ croît avec la pente. Ainsi, une vague donnée en eau profonde aura une plus forte valeur de $H_{b} / d_{b}$ sur une pente raide que sur une pente douce.

Weggel a cherché à ajuster une série de droites enveloppes pour chacune des pentes, en posant :

$$
\frac{H_{b}}{d_{b}}=b(\mathrm{~m})-a(\mathrm{~m}) \frac{H_{b}}{T^{2}}
$$

Dans cette équation $b(\mathrm{~m})$ est adimensionnel et $a(\mathrm{~m})$ en $s^{2} / \mathrm{m}$.

Les formes des fonctions d'approximation ont été choisies en fonction de leur asymptote supposée.

$\mathrm{Si}$ :

$$
\mathrm{m} \rightarrow 0\left\{\begin{array}{l}
b(\mathrm{~m}) \rightarrow 0,78 \\
a(\mathrm{~m}) \rightarrow 0
\end{array} \text { et } \frac{H_{b}}{d_{b}} \rightarrow 0,78\right.
$$

si $m \rightarrow \infty$ (mur vertical), on prend pour valeur limite 2 fois 0,78 (somme de l'amplitude incidente et de l'amplitude de la houle parfaitement réfléchie).

Alors :

$$
\begin{aligned}
& a(\mathrm{~m})=4,46\left(1-e^{-19 \mathrm{~m}}\right)\left(s^{2} / \mathrm{m}\right) \\
& b(\mathrm{~m})=\frac{1}{0,64\left(1+e^{-19,5 \mathrm{~m}}\right)}
\end{aligned}
$$

En combinant (25), (26), (27), on peut déterminér $H_{b} / d_{b}$ pour n'importe quelles pente de plage et cambrure de déferlement.

Les droites ajustées sont représentées sur la figure 14 ainsi que leur enveloppe.

Les valeurs de la hauteur de déferlement définies selon le cas par (25), (26) et (27) correspondent au point où le déferlement commence.

On suppose que le plus grand déferlement frappant la structure est celui qui se produit à une distance $x_{b}$ égale à $\varphi x_{p}, x_{p}$ étant la distance de plongée d'un déferlement plongeant.

Si $d_{s}$ est la profondeur d'eau au pied de la structure, $d_{b}$ la profondeur critique de déferlement, on a pour des raisons géométriques les relations :

$$
d_{s}=d_{b}-\mathrm{m} x_{b}
$$

soit adimensionnellement :

$$
\frac{d_{s}}{H_{b}}=\frac{d_{b}}{H_{b}}-\mathrm{m} \frac{x_{b}}{H_{b}}
$$

or, d'après (26) :

$$
\frac{x_{b}}{H_{b}}=\frac{\varphi x_{p}}{H_{b}}=\varphi \tau_{p}=\varphi(4-9,25 \mathrm{~m})
$$


La résolution du système d'équation (25), (26), (27), (29), (30) conduit à :

$$
\begin{aligned}
& \frac{H_{b}}{d_{s}}=\left[\frac{1}{m a \varphi(18,5 \mathrm{~m}-8)}\right]\left\{a+\frac{1}{\frac{d s}{T^{2}}}\left(1+9,25 \mathrm{~m}^{2} b \varphi-4 \mathrm{~m} b \varphi\right)\right. \\
& \left.-\sqrt{\left[a+\frac{1}{\frac{d s}{T^{2}}}\left(1+9,25 \mathrm{~m}^{2} b \varphi-4 \mathrm{~m} b \varphi\right)\right]^{2}-\frac{4 m b a \varphi}{\frac{d s}{T^{2}}}(9,25 \mathrm{~m}-4)}\right\}
\end{aligned}
$$

Weggel suppose que la distance $d$ à laquelle se produit le déferlement le plus dangereux est égale à $x_{b}$. Soit $\varphi=1$.

La figure 16 représente la relation entre $H_{b} / d_{s}$ et $d_{s} / T^{2}$ pour différentes pentes de plage dans cette hypothèse.

Pour une hauteur $H_{b} / T^{2}$ déterminée, la formule (13) permet alors de déterminer le creux de la houle non réfractée en eau profonde par approximations successives.

Pour notre part, nous ne retenons pas à priori l'hypothèse de Weggel selon laquelle le déferlement le plus dommageable pour une structure est celui qui se produit à une distance égale à la distance de plongée de la structure.

Supposons :

$$
0 \leqslant \varphi \leqslant 2
$$

Nous essayerons à l'aide des essais réalisés par SOGREAH de déterminer $\varphi$.

\section{II - Rappel des connaissances actuelles sur les ouvrages en blocs}

Nous ne traiterons ici que du cas des ouvrages, digues, perrés, épis à talus, en blocs naturels ou artificiels.

Nous supposerons également généralement que l'attaque de la houle est frontale.

Lorsque la transposition sera simple, entre le cas de la houle oblique et de la houle frontale, elle sera indiquée.

\section{1 - ACTION D'UNE LAME DEFERLANTE SUR UN OU- VRAGE A TALUS EN BLOCS}

1.1 - Une structure en blocs du type couramment employé pour la protection de la houle est composée d'un noyau de blocs de formes quelconques placés diversement et protégés par une carapace d'enrochements choisis ou par des blocs de béton de formes spéciales.

Une structure en blocs peut être définie par plusieurs caractéristiques. Pour chacune des couches :

- la dimension des blocs caractérisée par une dimension linéaire,

- la forme des blocs,

- l'orientation des blocs,

- la rugosité absolue,

- la porosité.

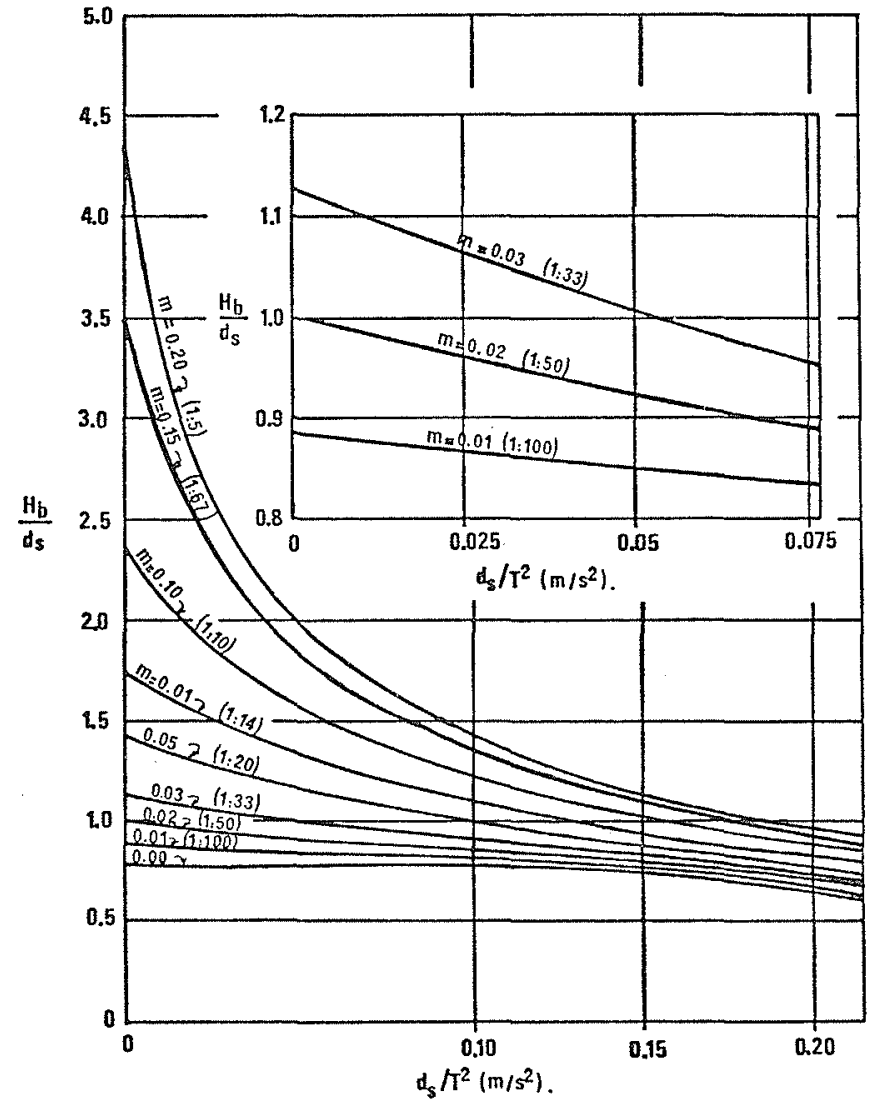

16/ Hauteur maximum adimensionnelle d'un déferlement $\frac{H_{b}}{d_{s}}$ fonction de la profondeur au niveau de la structure $\frac{d_{s}}{T^{2}}\left(\mathrm{~m} / \mathrm{s}^{2}\right)$ (D'après Weggel).

Selon Hudson [27] la porosité d'un brise-lames de blocs déversés pêle-mêle varie de $39 \%$ à $47 \%$ selon la forme des enrochements.

De même on démontre que la stabilité des blocs est maximum lorsqu'un grand axe est perpendiculaire au talus.

\section{2 - Mécanisme de l'action de la houle sur une digue}

On peut distinguer deux masses d'eau bien distinctes, l'une à l'extérieur suivant les mouvements de la houle, l'autre à l'intérieur de la carapace et de la sous-couche se déplaçant avec un certain déphasage avec la masse d'eau extérieure (figure 17).

La limite entre $V$ int et $V$ ext est située dans le cas d'une carapace en cubes à mi-épaisseur de la carapace.

Si l'on appelle $T_{c}$ la période de coupure de la digue, c'est-àdire la période d'une houle non déferlante pour laquelle les mouvements de $V$ int et $V$ ext sont en phase : pour une fréquence supérieure à $1 / T_{c}$ les variations du niveau de l'eau à l'extérieur ne sont transmises qu'avec un certain retard et avec une amplitude moindre à l'intérieur de la carapace.

1.2.1 - Si $T \gg T_{c}$, l'arrivée d'une seconde lame se produit lorsque l'intérieur de la carapace ne s'est pas vidé (figure 18).

La pénétration au sein de la carapace s'en trouve contrariée et les blocs sont relativement peu sollicités. 


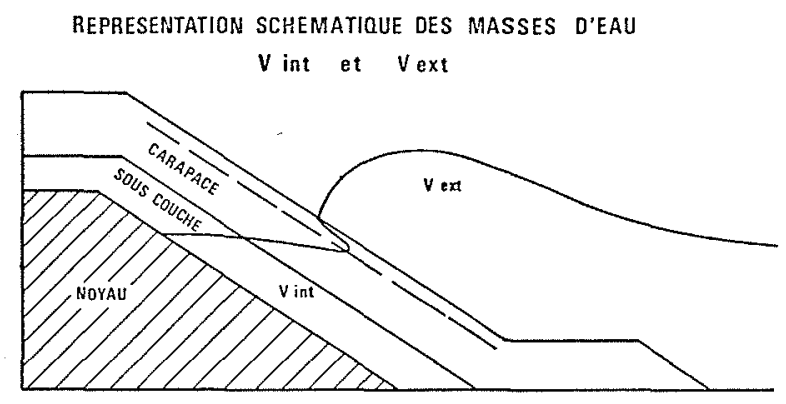

$17 /$

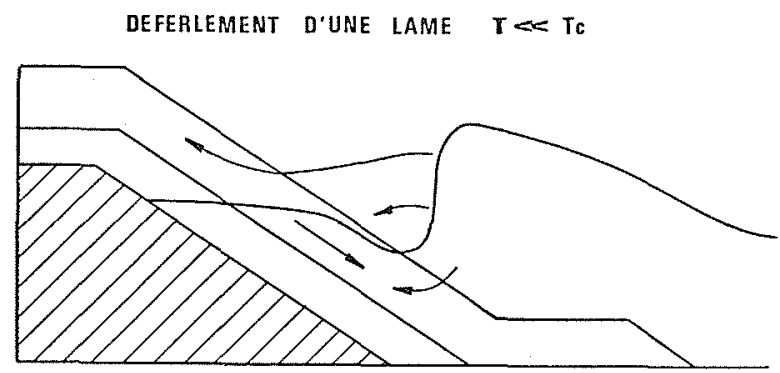

$18 /$

DEFERLEMENT POUR $T \geqslant T \mathbf{c}$

(En pointillés. situation favorisée par un cavalier trop imperméable)

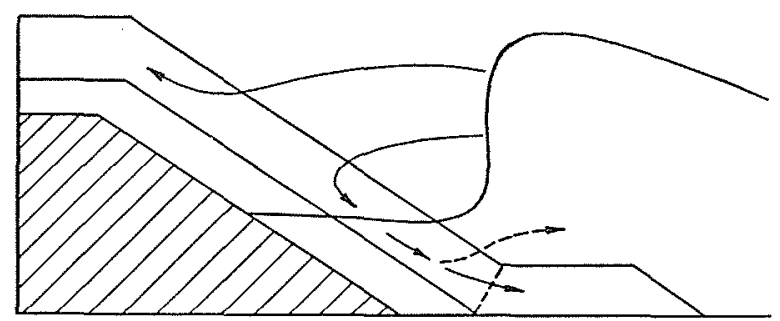

$19 /$

1.2.2 - Si $T \ll T_{c}$ (voir figure 19), l'arrivée du front raide correspondant à la lame déferlante a lieu alors que la carapace est vide d'eau.

Il y a d'abord pénétration à l'intérieur de la digue, puis dissociation de la masse d'eau incidente, $V_{H}$ vers le haut et $V_{B}$ vers le bas.

L'éclatement de la lame tend à ouvrir la carapace à un niveau moyen durant le reflux. Si le cavalier de pied est trop peu perméable $V_{B}$ aura rapidement tendance, en début de reflux à rejaillir au-dessus du cavalier.

1.3 - Influence du type de déferlement sur la stabilité des structures en blocs.

J.P. Ahrens [26] a effectué des essais systématiques en cuve à houle de très grandes dimensions sur une digue en enrochements ; ils ont permis de mettre en évidence divers types d'attaque d'une structure en blocs selon le type de déferlement.

1.3.1 - Le déferlement plongeant frappe les blocs de la carapace avec un grand impact presque normal à la carapace et donc avec une faible composante parallèle à cette carapace.
Par ailleurs, l'ascension suivant la plongée du déferlement concerne une masse composite d'air et d'eau et entraîne un faible impact sur les blocs.

Le reflux n'a pas le volume d'eau suffisant pour développer une force considérable.

1.3.2 - En cas de déferlement gonflant, la lame s'élève le long du talus et l'impact est faible. Par contre, le reflux est dangereux en soulevant des blocs à l'extérieur de la carapace.

1.3.3 - Le déferlement avec effrondrement est le plus dangereux.

L'ascension semble exercer un impact significatif contre les blocs tangentiellement à la pente. Le reflux peut alors déplacer les blocs rendus instables lors de l'ascension.

Faute d'un accord entre les auteurs sur un critère caractérisant le déferlement avec effondrement, nous avons suivi la méthode de Weggel qui aboutit à inclure la plus grande partie de ces déferlements parmi les déferlements plongeants, et parmi les déferlements gonflants.

\section{2 - RUINE D'UNE STRUCTURE EN BLOCS SOUMISE A UN DEFERLEMENT}

2.1 - Les modes de destruction d'un ouvrage soumis à déferlement ne sont pas différents des modes de destruction des ouvrages soumis à des houles non déferlantes.

Rappelons que les modes de destruction d'une structure en blocs sont :

- le glissement lorsqu'une section de la structure devient instable et glisse le long de la face avant de cette structure ;

- le soulèvement lorsque des blocs individuels sont projetés directement hors de cette structure ;

- la destruction par impact lorsque l'impact de la lame déferlante pousse ou roule les enrochements de couverture au-dessus du couronnement de la structure.

La ruine par glissement se produit si les forces parallèles au talus et dirigées vers le bas excédent la résistance au frottement entre les blocs et leur support. Si cette instabilité est localisée les blocs adjacents fournissent la réaction nécessaire pour s'opposer au mouvement; si elle se produit simultanément sur une section du brise-lames, cette section peut glisser vers le bas.

La ruine par soulèvement se produira si les forces normales positives s'exerçant sur un bloc excédent la composante normale du poids du bloc. Dans ce cas le frottement sera faible.

Cette condition est généralement limitée à un bloc. Cependant, le glissement des blocs supérieurs à cet emplacement peut alors produire une ruine progressive.

La ruine par impact se produit si le brise-lames a une capacité insuffisante pour fournir la réaction nécessaire contre les forces parallèles au talus, importantes et causées par. l'impact. Dans ce cas, les forces d'impact vont pousser ou rouler les blocs au-dessus du brise-lames.

2.2 - Il ressort de ceci que deux cas de stabilité doivent être envisagés pour chaque bloc. La stabilité parallèle à la pente et la stabilité perpendiculaire à la pente. D'après Roald Svee [4] le cas de glissement vers le bas est le plus critique pour des pentes plus raides que $1 / 2,5$. 


\section{3 - Butée de pied et risberme de protection}

L'action de la houle décroissant avec la profondeur, il est habituel de ne pas prolonger la carapace jusqu'au pied de l'ouvrage. Au-dessous de la carapace peuvent donc être disposées des couches d'enrochements plus petites prolongeant les couches intermédiaires du filtre. Si le talus côté mer de cette butée est en avant du talus de la carapace on dispose d'une risberme horizontale (voir figure 20 ).

Dans les zones de fond horizontal, la risberme est conçue comme un artifice permettant par une forte dissipation de l'énergie d'utiliser des blocs de taille moindre. Cet intérêt a souvent été discuté.

L'intérêt des risbermes dans le cas de fonds en pente est de constituer un blocage permettant d'éviter l'effet d'un glissement massif des blocs sur les fonds.

Beaudevin en 1954, lors d'essais sur une digue à talus en enrochements homogène, a trouvé que le fond le plus bas à partir duquel les enrochements ne sont plus sollicités par la houle est situé à une profondeur égale à $1,3 \mathrm{H}$, le creux de la vague ne descendant jamais à cette cote.

Barailler en 1957 a situé cette distance à $1,2 \mathrm{H}$.

Inversement Sigurdsson en 1962 [3] a découvert au cours d'essais que pour la pente $1 / 1$ les points des maxima des courbes d'effort normal sont généralement situés à plus d'une hauteur de vague en-dessous du niveau de l'eau au repos. Il en concluait donc que "la pratique de réduire la dimension des blocs situés à plus d'une hauteur de vague en-dessous du niveau de l'eau au repos peut se révéler dangereuse".

Cette conclusion n'est d'ailleurs pas en opposition formelle avec les travaux de Beaudevin et Barailler.

La conception de la butée de pied relève donc encore beaucoup de l'empirisme. Il ne fait pas de doute néanmoins que la stabilité du pied de carapace empêche les mouvements dans la direction de la pente.

\section{3 - MODE DE CALCULS DES OUVRAGES A TALUS EN BLOCS}

De très nombreuses formules existent pour déterminer la stabilité des ouvrages en enrochements soumis à la houle, d'Irribaren à Hudson avec parmi les auteurs français celles de Larras et Beaudevin.

Il s'est néanmoins révélé impossible jusqu'à ce jour de déterminer par des méthodes analytiques, les conditions de déplacements des blocs de la carapace. Ces formules de stabilite sont done largement empiriques, mais constituent néln. moins un guide utile atu projeteur.

Seule la formule d'Hudson :

$$
W=\frac{\rho_{u} H^{3}}{K_{D}(\delta-1)^{3}} \frac{1}{\operatorname{Cotg} \alpha}
$$

avec :

$\rho_{a}$ masse volumique du bloc.

$\delta$. densité relative du bloc par rapport à l'eau.

$K_{D}$ indice de stabilité.

a été adaptée au cas du déferlement par une réduction du coefficient $K_{D}$ dans le cas de deferlement (cf. Tableau fig. 21).

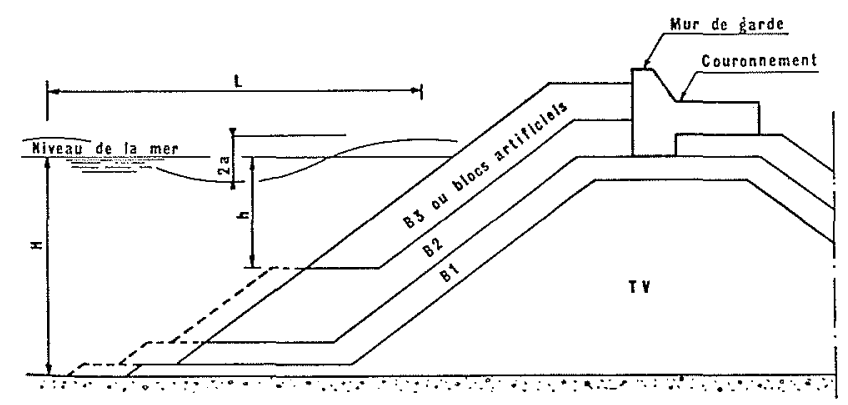

20/Digue à talus. Butée de pied de la carapace

Dans ces formules $H$ est le creux (ou l'amplitude) de la houle de projet.

Le poids calculé est le poids nécessaire pour n'obtenir aucun dommage pour cette houle de projet.

On ne conseille pas d'employer des enrochements de granulométrie continue dès que les hauteurs de houle excèdent $1,5 \mathrm{~m}$. Les formules de Hudson conduisent dans ce cas à des poids considérables.

Les valeurs de $K_{D}$ dans le cas de déferlement sont des formules prudentes conduisant à des poids plus élevés que ceux obtenus cas par cas en Laboratoire.

Ahrens [26] a établi pour des essais sur une digue en enrochements tout venant de pente 2,5/1 que le coefficient de stabilité de la formule d'Hudson variait selon le type de déferlement, d'une valeur de l'ordre de 6 pour des déferlements gon-

\begin{tabular}{|c|c|c|c|c|c|}
\hline \multicolumn{6}{|c|}{ - Valeurs de $K_{D}$ de la formule d'Hudson } \\
\hline \multirow[b]{2}{*}{$\begin{array}{l}\text { Nature des blocs } \\
n(\mathrm{a})\end{array}$} & \multirow[b]{2}{*}{$\begin{array}{l}\text { Mise en } \\
\text { place }\end{array}$} & \multicolumn{2}{|c|}{ Corps de la structure } & \multicolumn{2}{|c|}{ Musoir } \\
\hline & & $\begin{array}{l}\text { Houle dé- } \\
\text { ferlante } \\
\text { (b) }\end{array}$ & $\begin{array}{l}\text { Houle non } \\
\text { déferlante } \\
\text { (c) }\end{array}$ & $\begin{array}{l}\text { Houle dé- } \\
\text { ferlante } \\
\text { (b) }\end{array}$ & $\begin{array}{l}\text { Houle non } \\
\text { déferlante } \\
\text { (c) }\end{array}$ \\
\hline $\begin{array}{l}\text { Enrochements arrondis } \\
\text { réguliers }\end{array}$ & au hasard & 2.5 & 2.6 & 2.0 & 2.4 \\
\hline $\begin{array}{l}\text { Enrochements arrondis } \\
\text { réguliers } \quad 3\end{array}$ & $"$ & 3.0 & 3.2 & - & 2.9 \\
\hline $\begin{array}{l}\text { Enrochements grossiers } \\
\text { aux arêtes vives } 1\end{array}$ & $"(d)$ & 2.3 & 2.9 & 2.0 & 2.3 \\
\hline $\begin{array}{l}\text { Enrochements grossiers } \\
\text { aux aretes vives } \quad 2\end{array}$ & $"$ & 3.0 & 3.5 & 2.7 & 2.9 \\
\hline $\begin{array}{l}\text { Enrochements grossiers } \\
\text { aux aretes vives } \quad 3\end{array}$ & $"$ & 4.0 & 4.3 & - & 3.8 \\
\hline $\begin{array}{l}\text { Enrochements grossiers } \\
\text { aux arêtes vives } \quad 2\end{array}$ & Spécial (e) & 5.0 & 5.5 & 3.5 & 4.5 \\
\hline Cube modifié & au hasard & 7.0 & 7.5 & - & 5.0 \\
\hline Tétrapode & $"$ & 7.5 & 8.5 & 5.0 & 6.5 \\
\hline Quadripode & $"$ & 7.5 & 8.5 & 5.0 & 6.5 \\
\hline Hexapode & $"$ & 8.5 & 9.0 & 5.0 & 7.0 \\
\hline Tribarres & $"$ & 8.5 & 10.0 & 5.0 & 7.5 \\
\hline Tribarres & uniforme & 12.0 & 15.0 & 7.5 & 9.5 \\
\hline $\begin{array}{l}\text { Enrochements à granu- } \\
\text { tométrie continue }\end{array}$ & au hasard & $K_{R R}$ & $\begin{array}{r}1.7 \text { si la } p \\
6 \mathrm{~m} \text { et } 1.3 \\
\text { deur }\end{array}$ & $\begin{array}{l}\text { rofondeur } \\
6 \mathrm{~m} \text {. }\end{array}$ & \\
\hline \multirow{4}{*}{\multicolumn{6}{|c|}{$\begin{array}{l}\text { (a) n est le nombre de blocs par couches. } \\
\text { (b) Critère de faible submersion. } \\
\text { (c) Critère de non submersion. } \\
\text { (d) L'utilisation d'une seule couche d'enrochements est déconseillée sauf en cas de condition spéciale } \\
\text { et lorsqu'elle est utilisée, l'enrochement doit être soigneusement placé. }\end{array}$}} \\
\hline & & & & & \\
\hline & & & & & \\
\hline & & & & & \\
\hline \multicolumn{6}{|c|}{$\begin{array}{l}\text { (e) Se réfère à un placement spécial avec l'axe longitudinal du rocher placé normalement à la face de } \\
\text { la structure. }\end{array}$} \\
\hline
\end{tabular}
$21 /$ 


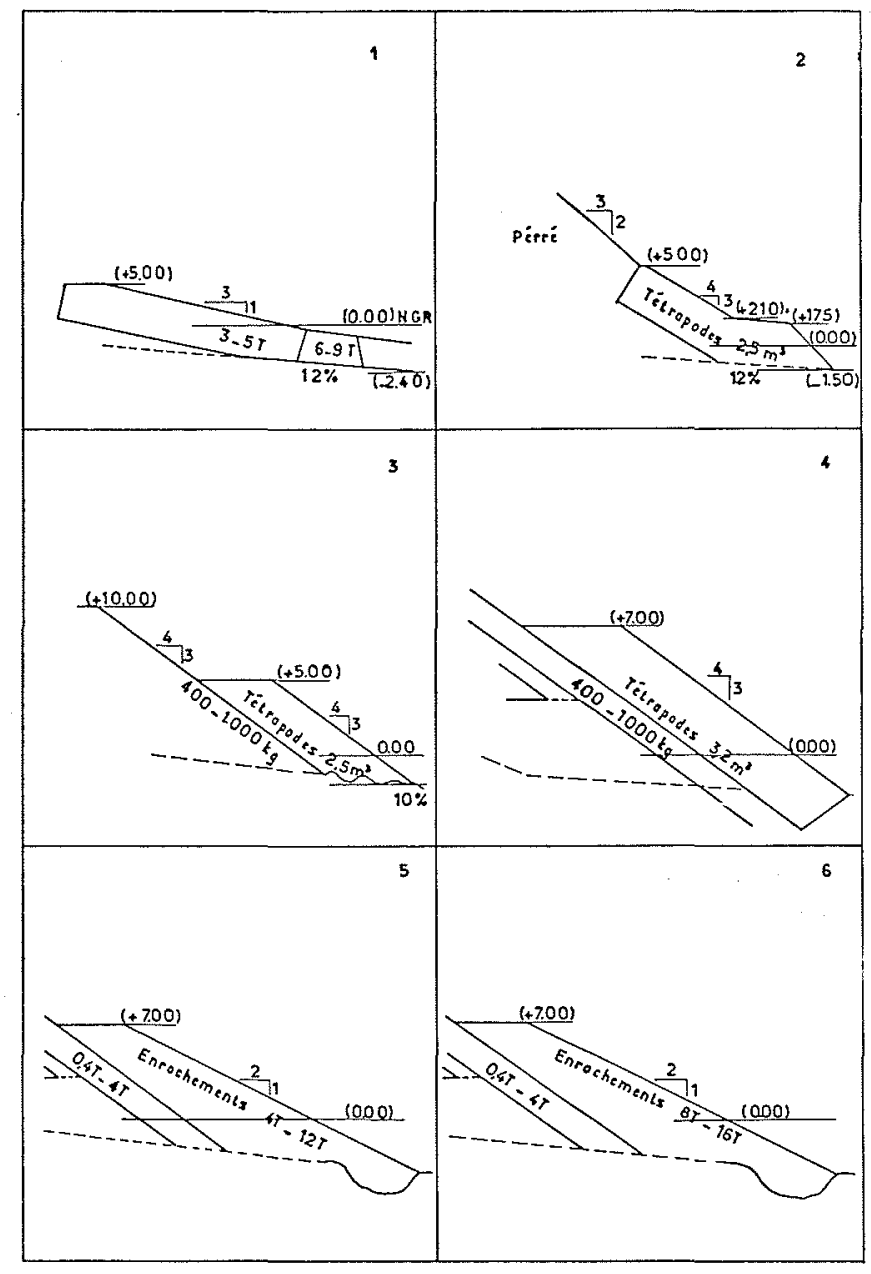

$22 \mathrm{a} /$

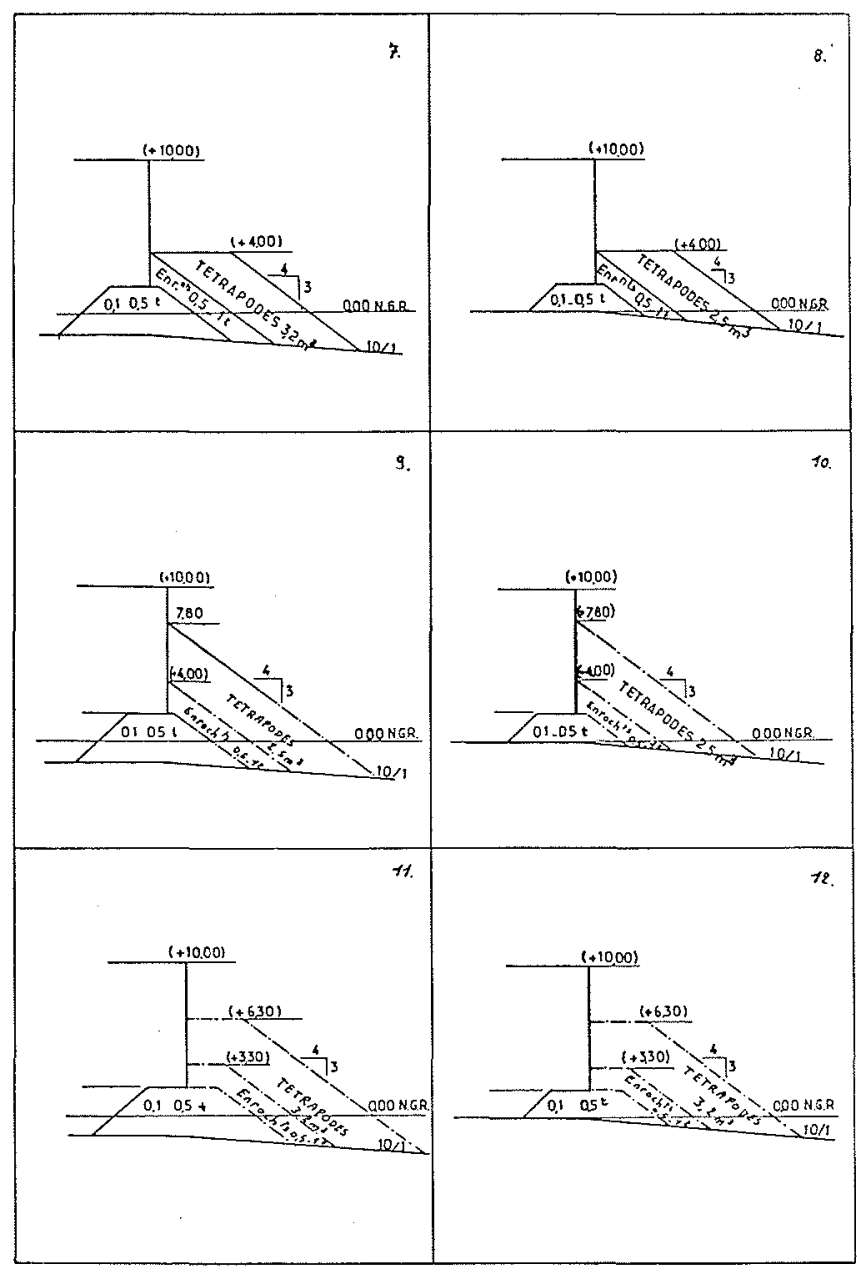

$22 \mathrm{~b} /$ flants et plongeants, à une valeur voisine de 2,5 pour des déferlements avec effondrement.

Il semblerait donc que les valeurs données par Hudson correspondent au cas de déferlement le plus défavorable, avec en outre une certaine sécurité.

\section{III - Méthode de calcul des ouvrages situés dans la zone de déferlement des houles}

1 - L'exposé ci-dessus suggère, malgré la dispersion des résultats ayant conduit aux diverses formules utilisées, une méthode de calcul des ouvrages dans la zone de déferlement des houles, en déterminant une houle de projet que l'on introduirait dans une formule genre Hudson ou Barailler pour déterminer le poids des blocs de la carapace et de la butée de pied.
Pour tester la valeur de cette méthode, nous avons repris des essais effectués par SOGREAH en vue de la mise au point de la défense d'une route dans le Département de la Réunion.

Ces travaux consistaient à élargir sur la mer la route en pied de falaises hautes de $70 \mathrm{~m}$ en moyenne, qui relie St-Denis-dela-Réunion au Port de la Pointe-des-Galets.

Cet élargissement était réalisé par remblaiement sur une plateforme d'abrasion peu profonde, de $100 \mathrm{~m}$ de large environ.

Le site des travaux était à une profondeur d'environ de (-3). L'étude devait révéler que la pente de cette zone d'abrasion était voisine de $10 \%$. En outre, des cyclônes passant tous les étés au voisinage de la Réunion provoquent des houles de très grande période 9 à $13 \mathrm{~s}$ et des surcotes de mer pouvant atteindre $0,80 \mathrm{~m}$. Les talus de protection de cette route sont donc situés dans la zone de déferlement des houles de tempête, ce qui a rendu sa construction extrêmement délicate.

En l'absence de théorie établie, des essais furent confiés à SOGREAH de 1970 à 1974 pour définir la protection de cette route. A cette occasion, SOGREAH testa une vingtaine de types de protection différents, dans des conditions diverses de pente des fonds, de profondeur, d'angle d'incidence de la houle (figures 22a, 22b, 22c). 


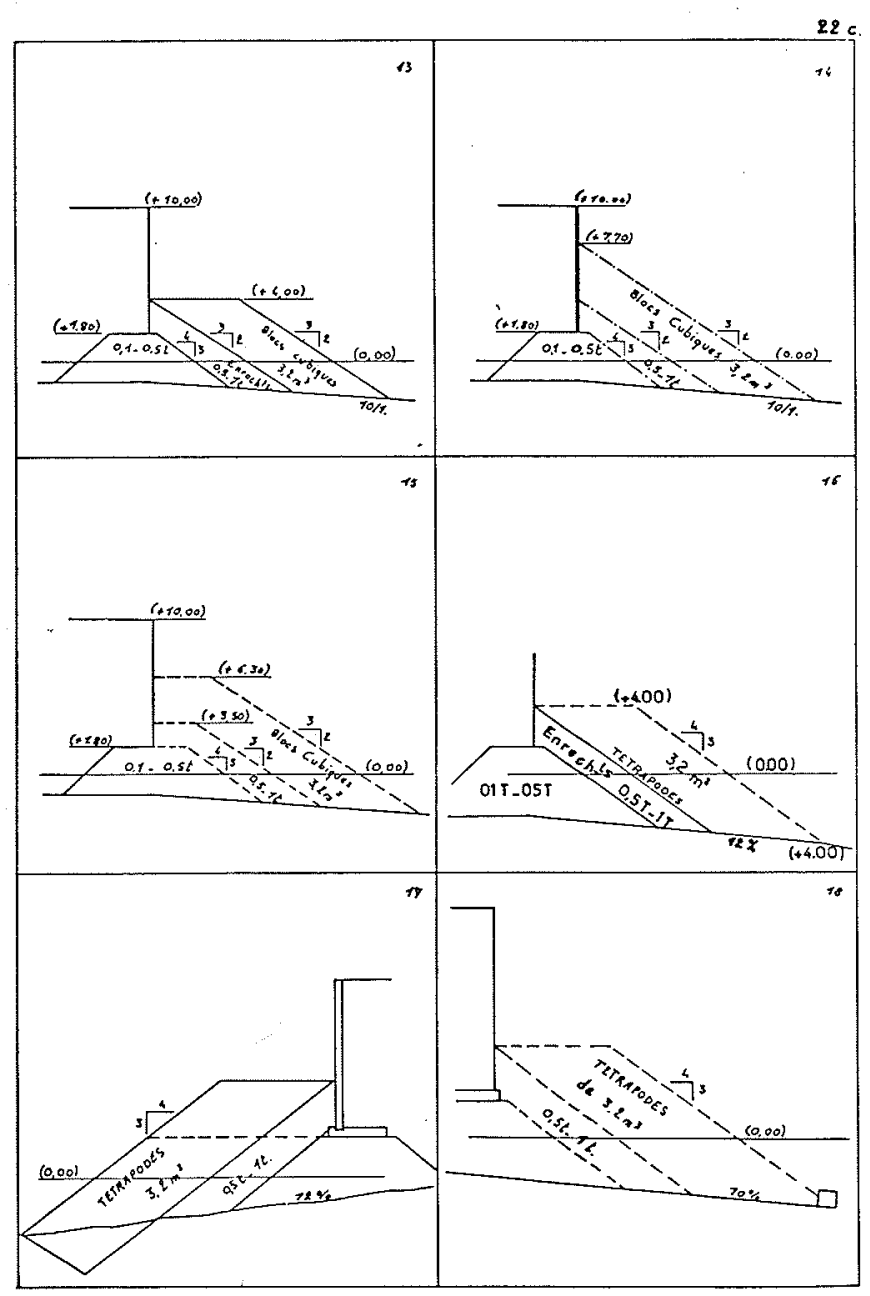

$22 \mathrm{c} /$

Ces essais très disparates ne pouvaient, et ce n'était pas le but recherché, conduire à établir une doctrine sur la construction des ouvrages en zone de déferlement.

Cependant, ils pouvaient être utiles pour ajuster une méthode empirique de calcul.

\section{2 - PRINCIPE DE LA METHODE DE CALCUL}

\section{1 - Calcul de la houle de projet}

Les hypothèses sont :

$m$ : la pente de la plage.

$T$ : la période de la houle.

$d_{s}$ : la profondeur d'eau en pied de structure au repos.

$\alpha \quad$ : l'angle de talus avec l'horizontale.

$K_{D}$ : l'indice de stabilité de la formule d'Hudson.

$\delta \quad$ : la densité de matériaux.

On calcule successivement $H_{b}$, amplitude de déferlement à différentes distances de la structure en faisant varier $\varphi$ de 0.10 en 0.10 entre 0 et 2 . Cette méthode permet de faire varier la distance de deferlement. Le déferlement se produit près du pied de structure pour $\varphi=0$ et à très grande distance de la structure pour $\varphi=2$.
Par les formules données (figure 9) on classe les déferlements en déversant, plongeant, gonflant. Puis, on détermine la cote du sommet du déferlement en supposant que le déferlement s'élève au-dessus du niveau moyen de $0,7 \mathrm{H}$ (en réalité $0,55 H$ à $0,85 H$ ).

On détermine également $H_{b} / T^{2}$ qui permet de déterminer si l'on utilise la partie rectiligne ou l'enveloppe des courbes de la figure 14.

On calcule ensuite la profondeur et la distance du déferlement.

Une itération de (13), (14), (15), (16) permet de calculer $H_{0}^{\prime}$ avec $L_{0}=1,56 T^{2}$ ( $T$ en $\mathrm{s}, L_{0}$ en $\mathrm{m}$ ).

\section{2 - Calcul du poids des blocs}

Avec $H=H_{b}$ dans la formule d'Hudson on peut alors calculer le poids des blocs nécessaires.

2.3 - Le même calcul a été fait compte tenu d'une súrélévation du niveau moyen de l'eau par rapport au niveau de l'eau au repos de $0,04 H_{b}$.

\section{3 - COMPARAISON AVEC LES ESSAIS}

3.1 - Sur les 52 dispositions étudiés par SOGREAH, 40 cas sont utilisables pour la comparaison.

Parmi ceux-ci, les 8 cas prévoyaient une risberme de pied, ils ont été traités comme les cas sans risberme.

Les 5 cas de houle inclinée ont été traités en tenant compte de la réfraction de la houle et en prenant pour pente $m$, la pente de la plage dans la direction de propagation de la houle.

3.2 - On a comparé successivement dans les deux cas : - en supposant le niveau moyen égal au niveau de l'eau au repos,

- en tenant compte d'une surélévation :

- la cote du sommet du déferlement comme indiqué cidessus avec la hauteur maximum d'ascension de la houle;

- le type de déferlement observé lorsqu'il était noté, au type de déferlement calculé ;

- l'amplitude du début de déferlement sur le pied de la carapace par $H_{0}^{\prime}$ calculé pour $\varphi=0$; ces trois comparaisons ayant pour but de tester la validité de la méthode de calcul ;

- la valeur de $\varphi$ correspondant à l'amplitude des dégât's maximum indiquée dans le rapport des essais ;

- la valeur de $\varphi$ correspondant au déferlement au-delà de la carapace sans effets sur l'ouvrage, selon les rapports des essais ;

- le poids du bloc calculé avec le poids du bloc utilisé (enrochements de poids uniforme, enrochements tout venant, cubes et tétrapodes) selon que l'essai concluait à la stabilité, à une stabilité limite ou à une instabilité de la carapace.

Une grande difficulté de cette comparaison résulte dans la difficulté d'interprétation des rapports d'essais : les essais étant effectués à amplitude de houle croissante de façon discontinue par sauts de l'ordre de $20 \%$, l'amplitude correspondant à un phénomène donné (début de déferlement sur la carapace) était donc déterminée avec une incertitude équivalente. 


\section{HOULE DEFERLANT AU PIED DE LA STRUCTURE}

\section{Calculs compte tenu de}

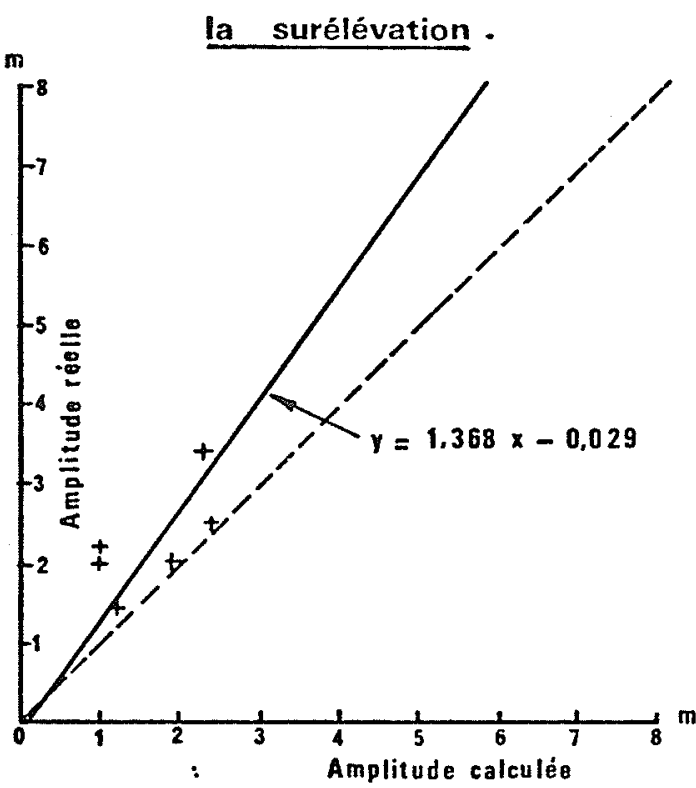

\section{Calculs compte non tenu}

de la surélévation -

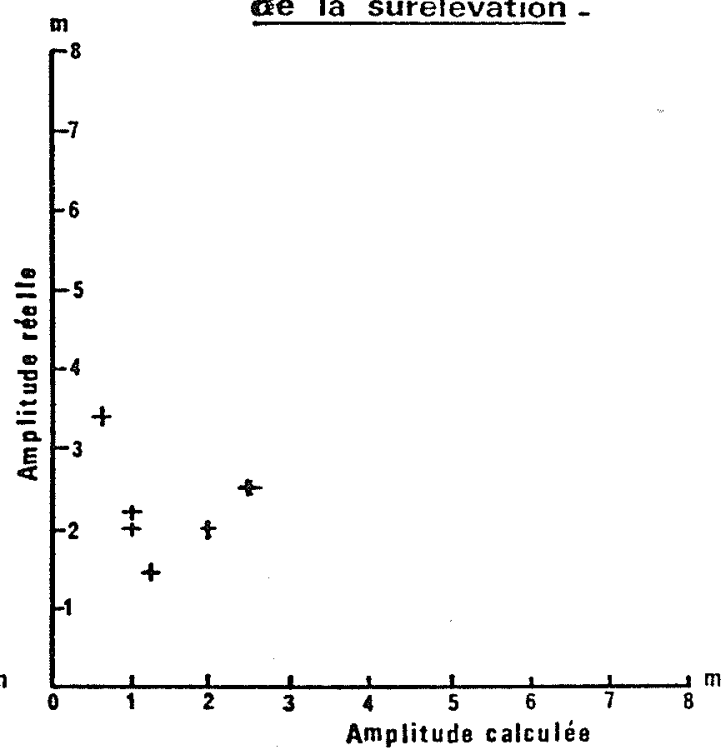

23a/ Déferlement gonflant,

Par ailleurs, le choix de l'amplitude de houle qualifié de "la plus dangereuse" était difficile à définir lorsque l'ouvrage était stable.

Lorsqu'il était instable, on choisissait généralement soit l'amplitude la plus élevée ayant entrainé des chutes de blocs, soit l'amplitude ayant provoqué la destruction complète de la carapace sans être assuré qu'une houle plus forte ne se serait pas révélée encore plus sévère (destruction plus rapide par exemple).

Ces considérations peuvent expliquer la dispersion des résultats.

\section{4 - RESULTATS DE LA COMPARAISON}

4.1 - Ascension maximum sur la digue et "cote" du sommet du déferlement provoquant les dégâts maximum ou provoquant des déferlements au-delà de la structure.

Aucune corrélation n'a pu être établie, la cote d'ascension maximum étant toujours inférieure à la "cote" calculée cidessous et restant constante à partir d'une certaine amplitude.

\section{2 - Type de déferlement observé et calculé}

Le type de déferlement était rarement indiqué, seulement lorsqu'un déferlement plongeant caractéristique était observé. Il y eut accord parfait entre les observations et le type de déferlement déterminé par le calcul.
4.3 - Comparaison entre l'amplitude observée du déferlement en pied de carapace et l'amplitude correspondant à $\varphi=0$. (Figures 23a 23b).

4.3.1 - Le calcul ne tient pas compte de la surélévation.

On obtient une très bonne corrélation dans le cas de déferlement plongeant $(r=0,91)$ et des corrélations médiocres en cas de déferlement déversant et gonflant,

en mètres, déferlement plongeant :

$$
H \text { réel }=1,102 H(\varphi=0)-0,129 \quad(r=0,91)
$$

en mètres, déferlement gonflant :

$$
H \text { réel }=1,368 H(\varphi=0)-0,029 \quad(r=0,68)
$$

en mètres, déferlement déversant :

$$
H \text { réel }=1,515 H(\varphi=0)-2,04 \quad-(r=0,71)
$$

Comme on le voit les amplitudes réelles de déferlement au pied de la structure sont légèrement supérieures aux amplitudes calculées.

4.3.2 - En tenant compte de la surélévation, la corrélation est beaucoup moins nette.

lin mètres, pour le déferlement plongeant :

$$
H \text { réel }=1,111 H(\varphi=0)-0,188 \quad(r=0,73) .
$$

Dans le cas de déferlement gonflant et déversant, aucune loi n'a pu être établie.

On observe un bon accord entre le calcul et l'expérience dans le cas de déferlement plongeant, ce qui se comprend aisé- 


\section{HOULE DEFERLANT AU PIED DE LA STRUCTURE}

\section{Calculs compte non tenu}

de la surélévation.

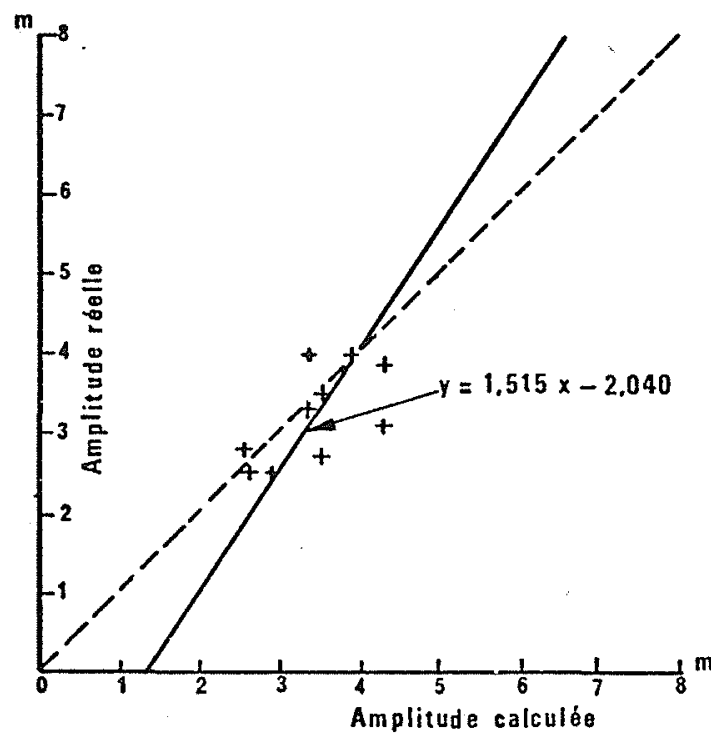

A DEFERLEMENT DEVERSANT
Calculs compte tenu de

la surélévation -

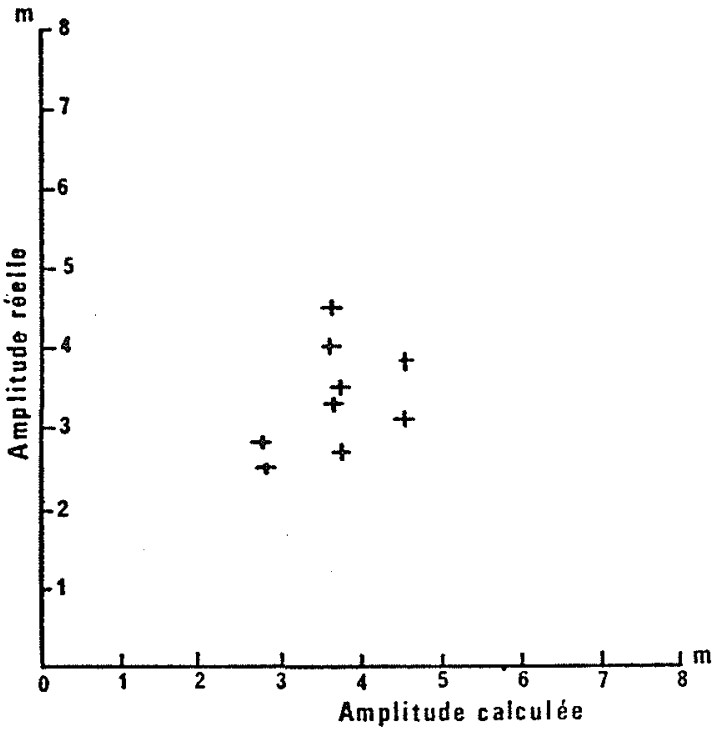

8/ DEFERLEMENT DEVERSANT

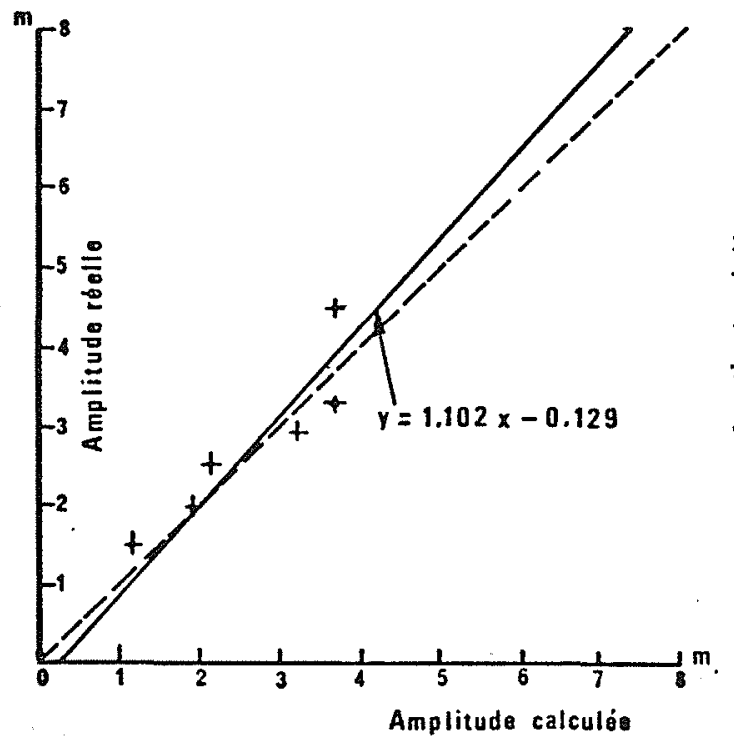

C/ DEFERLEMENT Plongeant

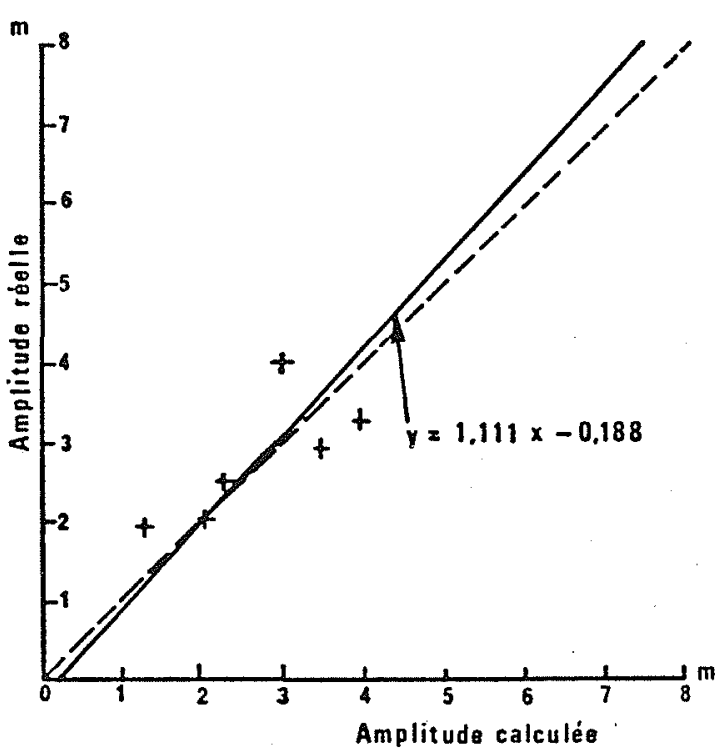

D/ DEFERLEMENT PLONGEANT

$23 b /$ 


\section{HOULE PROVOQUANT DES DEGATS MAXIMUM}

Calculs compte non tenu

de la surélévation.

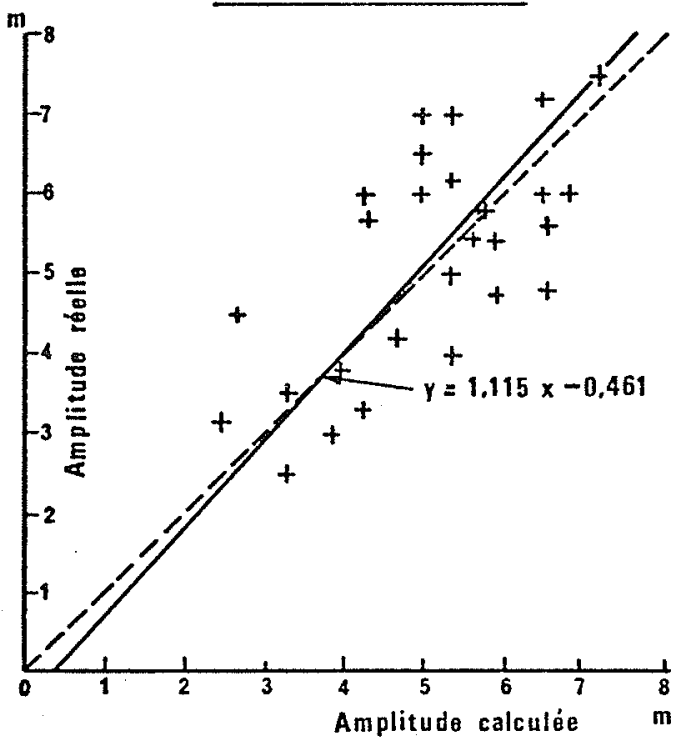

A/ DEFERLEMENT DEVERSANT

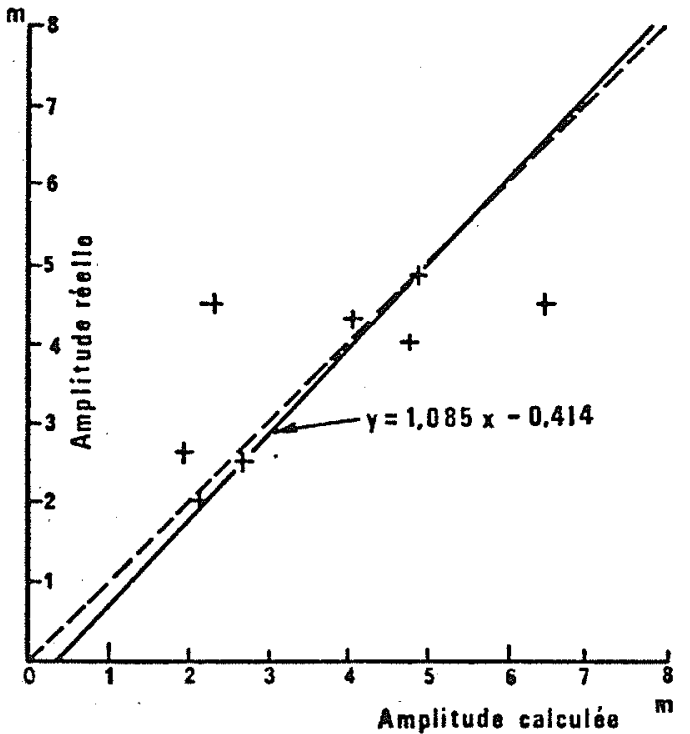

C/ DEFERLEMENT PLONGEANT
Calculs compte tenu de

la surélévation.

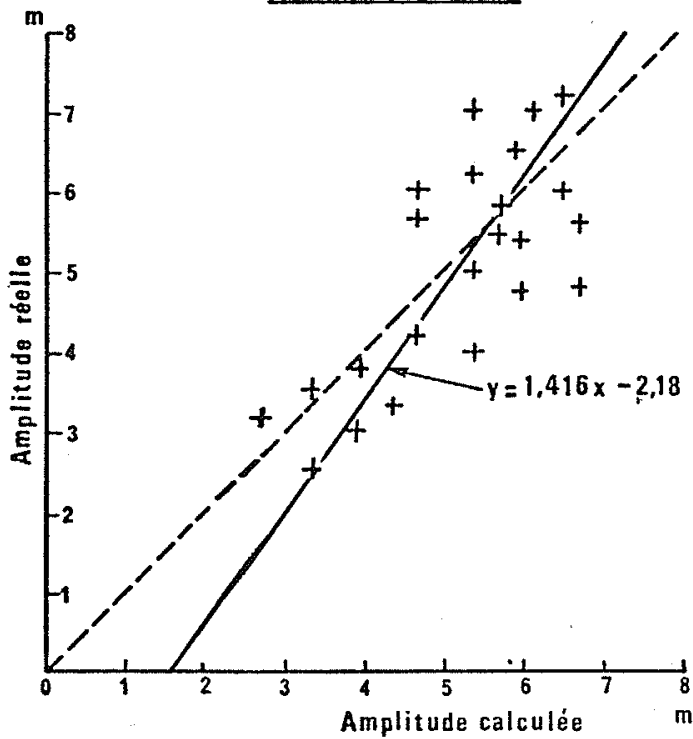

8/ Deferlement deVERSANT

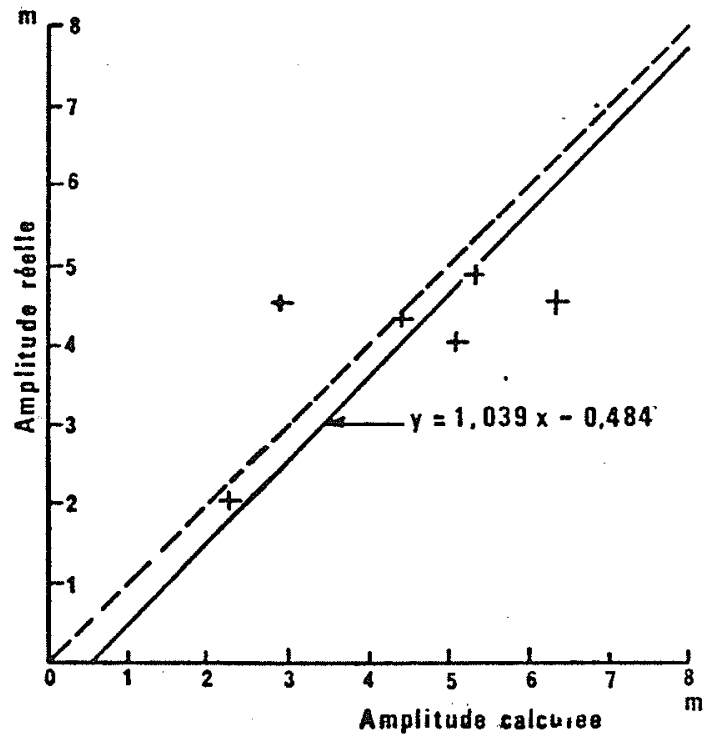

D/ DEFERLEMENT PLONGEANT 


\section{hOULE DEFERLANT AU-DELA DE LA STRUCTURE}

\section{Calculs compte non tenu}

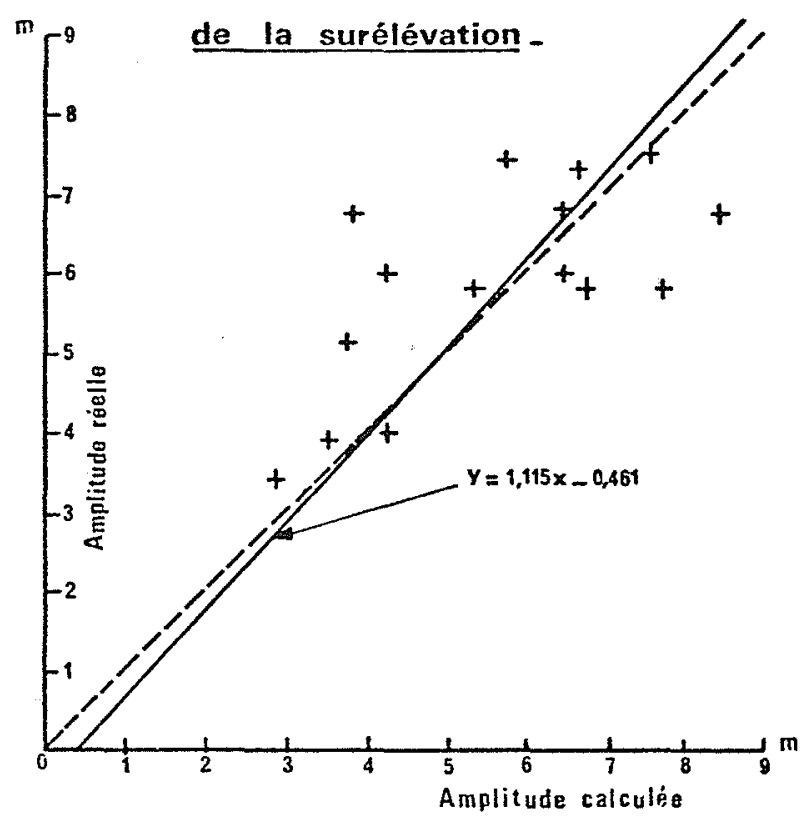

A/ DEFERLEHENT DEVERSANT

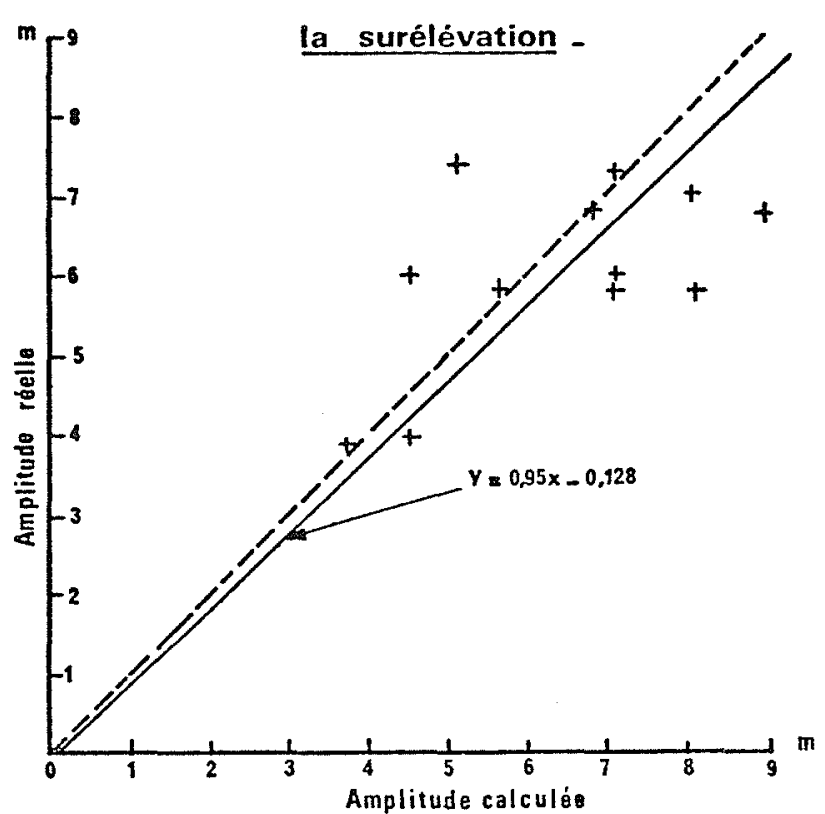

B/ DEFERLEMENT DEVERSANT ment étant donné que la distance de déferlement a été prise Egale dans tous les cas à celle du déferlement plongeint.

Dans ce cas, l'amplitude réelle est pratiquement égale à l'amplitude calculée.

Dans les autres cas, elle est nettement supérieure (voir figures $23 \mathrm{a}, 23 \mathrm{~b}$ ).

\section{4 - Valeur de $\varphi$ correspondant à l'amplitude de dégâts maxi- mum (figure 24).}

\subsection{1 - Calcul sans surélévation.}

Pour les déferlements déversants, la valeur de $\varphi$ correspondant aux dégâts maximum est en moyenne égale à :

1,06 avec un écart quadratique moyen de 0,37 .

Pour les déferlements plongeants :

1,07 avec un écart quadratique moyen de 0,28.

Les figures $24 a$ et $c$ montrent la corrélation entre les amplitudes réelles de la houle provoquant les dégâts maximum pour $\varphi=1,06$ dans le cas de déferlements déversants et $\varphi=1,07$ dans le cas de déferlements plongeants.

4.4.2 - Le même calcul a été établi dans le cas du calcul de l'amplitude tenant compte d'une surélévation. Dans ce cas, la valeur de $\varphi$ provoquant les déferlements maximum est égale à :

$-0,88$ pour les déferlements déversants, avec un écart quadratique moyen de 0,36 .

- 1,02 pour les déferlements plongeants, avec un écart quadratique moyen de 0,36 .
Les figures $24 \mathrm{~b}$ et d, montrent la corrélation entre les amplitudes réelles de la houle provoquant les dégâts maximum pour $\varphi=0,88$ dans le cas de déferlements déversants et $\varphi=1,02$ dans le cas de déferlements plongeants.

On constate que les déferlements plongeants les plus dommageables correspondent pour une structure donnée à des valeurs de $\varphi$ plus élevées que les déferlements déversants, ce qui signifie que la distance à laquelle ils sont le plus dangereux, donc l'amplitude du déferlement correspondant, est plus grande dans le cas de déferlement plongeant.

En l'absence d'essais en nombre suffisant aucune loi n'a pu être établie dans le cas de déferlements gonflants.

4.5 - Valeur de $\varphi$ correspondant à l'amplitude de la houle la plus faible déferlant au-ctela de la structure. sans effet sur cottedernicre

\subsection{1 - Calcul sans tenir compte de la surélévation.}

Cette houle se produisant par une profondeur importante, les déferlements sont essentiellement de type déversant.

Dans ce cas, $\varphi$ moyen $=1,49 \quad r=0,37$.

La figure 25 a représente la relation entre les valeurs de l'amplitude de la houle déferlante au-delà de la structure avec $\varphi=1,49$ et les amplitudes réelles.

4.5.2 - Le même calcul a été fait en tenant compte de la surélévation.

Dans le cas de déferlement déversant :

$$
\varphi \text { moyen }=1,42 \quad r=0,32
$$

Dans le cas de déferlement plongeant :

$$
\varphi \text { moyen }=1,67 \quad r=0,10
$$


La figure $25 b$, représente la relation entre les valeurs de l'amplitude de la houle déferlante calculée avec $\varphi=1,42$ et de la houle réelle.

Pour toutes ces valeurs, malgré la dispersion signalée plus haut, on note un bon accord entre les observations et les approximations ajustées.

\section{5 - CALCUL DES POIDS DES BLOCS}

5.1 - On a calculé par la formule d'Hudson, le poids des blocs correspondant à un creux de la houle au moment du déferlement de dégâts maximum calculé sans tenir compte de la surélévation.

On a comparé, dans trois cas, stabilité, stabilité limite et instabilité, le poids réel et le poids calculé.

On constate que dans le cas où la digue a été ruinée sous l'action de la houle, le poids calculé s'est révélé toujours supérieur au poids mis en place sauf dans un cas.

Un essai concernait une protection comprenant une risberme de pied, le déferlement étant plongeant. On voit donc que l'emploi de la formule d'Hudson peut dans ce cas conduire à des sous-estimations.

Dans les cas limites, le poids essayé est du même ordre de grandeur que le poids calculé, avec trois exceptions où le poids donné par la formule de Hudson s'est révélé très supérieur (déferlements déversants)

Dans le cas de stabilité, le poids calculé s'est révélé soit endessous, soit au-dessus du poids calculé. Dans le premier cas le déferlement itait plonguant, danș le second cas déversant.

Il semble donc que cette méthode donne les résultats voisins de ceux des essais dans le cas de déferlements plongeants, mais conduisent à surestimer le poids des blocs nécessaires en cas de déferlement déversant.

5.2 - Mêmes constatations dans le cas de calcul tenant compte de la surélévation avec des poids obtenus légèrement plus faibles.

Il semble donc que de tenir compte de la surélévation du niveau moyen de l'eau sur le niveau au repos n'apporte pas de précisions supplémentaires et entraîne une complication inutile du calcul.

\section{Conclusions}

La présente étude a permis de tester une méthode de calcul des ouvrages en enrochements situés dans les zones de déferlement face à une houle directe en fonction de la période de la houle, de la profondeur au pied de la structure, de la pente de la plage et de la nature de l'ouvrage.

Cette houle de projet introduite dans la formule d'Hudson permet de guider le choix des blocs de la carapace, de façon assez bonne dans le cas de déferlements plongeants ou gonflants. Elle tend à surdimensionner les blocs en cas de déferlement déversant.

La dispersion importante des résultats est néanmoins comparable à celle des essais en houle non déferlante.
Si la méthode exposée peut permettre de dimensionner de petits ouvrages, elle ne peut se substituer aux essais sur modèles pour les ouvrages de grandes dimensions. Elle peut permettre néanmoins de réduire le champ des investigations.

Elle donne d'assez bons résultats pour la détermination de la houle déferlante. Ces résultats sont moins satisfaisants pour le calcul du poids des blocs. Il semblerait, en effet, qu'il soit nécessaire d'adopter un indice de stabilité différent selon le type de déferlement.

Il reste à étudier particulièrement les déferlements avec effondrement, particulièrement destructeurs, dont le domaine d'existence recouvre partiellement celui des déferlements plongeants et gonflants, et que nous n'avons pas, dans notre étude, distingués de ces derniers.

Des recherches utiles s'imposent donc dans ce cas.

Pour sa part, la Direction des Ports Maritimes et des Voies Navigables a l'intention de faire entreprendre des essais systématiques sur la stabilité des ouvrages soumis au déferlement des houles.

\section{LISTE DES REFERENCES}

[1] Shore Protection Planning and Design. Technical Report $n^{\circ} 4$ U.S. Army CREC 1966.

[2] Shore Protection Manual. (U.S. Army. CREC 1973).

[3] G. SIGURDSSON. - "Wave Forces on Breakwater Capstones" Journal of the Waterways and Harbors Division. Proceedings of the American Society of Civil Engineers, Août 1962.

[4] Roald SVEE. - "Formulas for Design of Rubble-Mound Breakwaters" Journal of the Waterways and Harbors Division. Proceedings of the American Society of Civil Engineers, Mai 1962.

[5] GALVIN. - "Breaker Type Classification on three laboratory beaches" Journal of Geophysical Research, $\mathrm{n}^{\circ} 12,15$ Juin 1968

[6] GalviN. - "Breaker Travel and Choice of Design Wave Height" Journal of the Waterways Harbors Division. Proceedings of the $A S C E$, Mai 1969

[7] YUAN JEN and PANG-MOU LIN. - "Plunging Wave Pressures on Semicylindrical Tube" Journal of the Waterways and Harbors Division. Proceedings of the ASCE, Août 1971.

[8] Weggel. - "Maximum Breaker Height For Design" Proceedings of the 13th Coastal Enginering Conference, July 10-14 1972 Vancouver Canada.

[9] WEGGEL. - "Maximum Breaker Height" Journal of Waterways, Harbors and Coastal Engineering Division. Proceedings of the ASCE, Novembre 1972.

[10] Frederick E. CAMFIELD and R.L. STREET. - "Shoaling of Solidary Waves on Small Slopes" Journal of the Waterways and Harbors Division. Proceedings of the ASCE, Février 1969.

[11] J.A. BATTJES. - "Run-up Distributions of Waves Breaking on Slopes" Journal of the Waterways, Harbors and Coastal Engineering Division. Proceeding of the ASCE, Février 1971.

[12] Action de la houle sur la butée de pied de la carapace de protection. Etude SOGREAH 1974 pour le compte de la Direction des Ports Maritimes et des Voies Navigeables.

[13] MichE. - "Le pouvoir réfléchissant des ouvrages maritimes exposés à l'action de la houle" Annales des Ponts-et-Chaussées, Mai-Juin 1951 .

[14] LENAU, - "The Solitary Wave of Maximum Amplitude" Journal Fluid Mechanics. Vol. 26, No. 2, 1966, p. 309-320. 
[15] HARLOW et WELCH. - "Numerical calculation of time-dependent viscous incompressible flow of fluid with free surface", Phys. Fluids, 8, 2182, 1965

[16] HARLOW et al. - "Liquid waves by computer", Science, 149 , $1091,1966$.

[17] Mrche. - "Mouvements ondulatoires des mers en profondeur constante en décroissant" Annales des Ponts-et-Chaussees, 1944.

[18] COLLINS et WIER. - "Probabilities of Wave Characteristics in the Surf Zone" Tetra Tech Report $\neq$ TC-149, Sept. 1969

[19] IVERSEN. - "Waves and Breakers in Shoaling Water" Proceedings, Third Conference on Coastal Engineering, Berkeley, Californie, Octobre 1952.

[20] IVERSEN. - "Laboratory Study of Breakers" Gravity Waves, U.S. Bureau of Standards, Circular 52, 1952.

[21] WEGGEL et MAXWELL. - "Experimental Study of Breaking Wave Pressures" OTC-1244 Second Annual Offshore Technology Conference, Houston, Texas, Avril 1970.
[22] PATRICK et WIEgEL. - "Amphibian Tractors in the Surf" Proceedings, First Conference on Ships and Waves, Council on Wave Research and American Society of Naval Architects and Marine Engineers, 1955.

[23] GODA. - "A Synthesis of Breaker Indices" Transactions, Japanese Society of Civil Engineers, Vol. 2, Part 2, 1970.

[24] MUNCK. - "The Solitary Wave Theory and its Application to Surf Problems" Annals of the New Academy of Sciences, Vol. 51, Mai 1949.

[25] REID et BRETSCHNEIDER. - "Surface Waves and Offshore Structures" The Texas University, Technical Report $\neq 53-10$, Octobre 1953.

[26] P. AHRENS. - "The Influence of Breaker type on riprap Stability" Proceedings of twelfth Conference on Coastal Engineering, Washington 1970.

[27] HUDSON. - "Laboratory Investigations of Rubble-Mound Breakwaters", Journal of the Waterways and Harbors Division. Proceeding of the ASCE, Vol. 85, Septembre 1959, p. 93-121.

\section{Discussion}

Président : M. P. BERGERON

M. le Président remercie M. Tenaud et M. A. Graillot de leur intéressant travail. Il remercie ce dernier d'avoir réussi, dans le faible laps de temps qui lui était imparti, à résumer clairement un mémoire très complet en donnant les conclusions et sans s'appesantir sur les formules que chacun pourra retrouver dans le texte qui sera publié. Il ouvre ensuite la discussion.

Vous avez comparé, observe M. LEPETIT, les hauteurs de houle, calculées par votre méthode tenant compte de la pente du fond, avec celles déduites des essais effectués par SO.GRE.A.H. L'accord est bon, à la dispersion des résultats expérimentaux près. Avez-vous comparé les hauteurs observées avec celles calculées sans tenir compte de la pente du fond; l'écart obtenu est-il significativement supérieur?

Nous avons l'intention de reprendre cette comparaison en utilisant la formule de M. MicHE mais nous n'avons pas encore eu le temps de le faire, répond M. Graillot.

Les essais ont, dans une première phase, été effectués en canal, remarque $M$. Couprie. Les essais ayant pour objet l'étude de la protection de la route de La Réunion ont été effectués en bassin avec une incidence oblique de la houle à partir de 1974. L'analyse de M. Graillot prend-elle en compte les essais en bassin?

Les éléments qui viennent d'être exposés, précise M. TENAUD, ne concernent que des ouvrages perpendiculaires à la direction de la propagation de la houle. L'effet de l'obliquité de la houle est beaucoup plus important pour les houles déferlantes que pour les houles non déferlantes. Une houle oblique à $15^{\circ}$ est beaucoup plus agressive qu'une houle normale, comme l'ont montré les essais effectués pour la route littorale de La Réunion.

Donc, pour des essais sur modèle réduit sans houle déferlante d'ouvrages importants, il est quasi-indispensable d'opérer sur des modèles à trois dimensions (en bassin et non en canal).

Cette conclusion, valable pour les gros ouvrages, n'est-elle pas un peu pessimiste, observe $M$. le Président. Pour les petits ouvrages qui nous intéressent cet après-midi, pensez-vous que les valeurs approximatives que l'on peut tirer des formules données dans la communication puissent être utilisées sans faire des essais sur modèle réduit? Il y a, ici, des représentants des entrepreneurs de travaux publics; dans un tel cas, estiment-ils pouvoir utiliser ces formules approximatives?

M. Graillot pense qu'il s'agit là d'un problème économique; dans le cas de petits ouvrages, la formule de Hudson donne des résultats dans le sens de la sécurité; pour les grands ouvrages, il peut en résulter une augmentation inutile du coût et ce peut-être une raison pour recourir à des essais sur modèle réduit.
M. Couprie intervient ensuite en ces termes :

J'ai eu l'occasion d'étudier, à une certaine époque, les problèmes posés par les digues mixtes, qui sont des digues verticales placées au sommet d'un soubassement à talus. Or, les essais rapportés dans la littérature m'avaient révélé quelque chose d'assez surprenant: pour la même digue, sur la même embase, suivant que le fond a une pente de $1 / 50^{\circ}$ ou de $1 / 20^{\mathrm{e}}$ selon les cas, c'est l'une de ces pentes qui conduit aux chocs les plus violents sur l'ouvrage; le phénomène ne présente pas une continuité apparente; il y a une interaction entre la structure de la digue et le fond. On semble être en présence d'un phénomène de résonance; je n'ai pas trouvé de loi générale, mais j'ai observé des sortes de «pic »; pour un ouvrage déterminé, il existe une pente particulière qui conduit à un renforcement tout à fait exceptionnel de l'impact des lames; pour une faible variation en plus ou en moins de cette pente, le choc est beaucoup moins fort. Dans l'exposé qui a été fait, on retrouve en partie cette sorte de phénomène particulier. Comme dans ces digues mixtes on mesure en général les efforts de choc sur l'ouvrage luil-même, on a un critère quantitatif un peu plus simple que le critère de stabilité d'une digue à talus.

Le phénomène de "pic » dont vous parlez, dit M. GraIllot, pourrait peut-être s'interpréter comme l'apparition de déferlements avec effondrement qui sont les plus destructeurs de tous.

C'est l'intérêt des formules que j'ai données - formules dues à des auteurs américains tels que Weggel - de définir avec une bonne précision le domaine d'existence de ces "déferlements avec effondrement $»$.

Le profil réel du fond, précise M. TENAUd, a aussi une importance particulière pour le déferlement de la houle. Une pente moyenne peut ne pas toujours être représentative de la réalité. La rugosité des fonds joue aussi un grand rôle. Un fond très rugueux (présence de blocs de rochers comme à La Réunion) diminue l'énergie de la houle, donc l'amplitude maximale de celle qui peut atteindre l'ouvrage.

Il semble judicieux, estime $M$. Couprie, de rapprocher les observations faites, d'une part à propos de la défense des hauts de plage comme lors de l'étude de la défense de la route littorale de La Réunion et, d'autre part, sur les digues mixtes; dans les deux cas, il faut rechercher les déferlements les plus défavorables afin d'établir les ouvrages de protection en conséquence.

Je remercie, à nouveau, M. TENAUd et $M$. GRAILLOT, conclut M. le Président, et je donne la parole à M. LE Grand pour l'exposé de sa communication. 